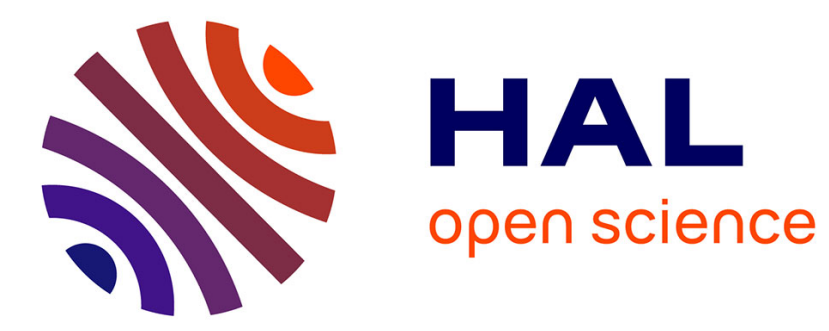

\title{
A Review of Learning Planning Action Models
}

Ankuj Arora, Humbert Fiorino, Damien Pellier, Marc M’Etivier, Sylvie

Pesty

\section{To cite this version:}

Ankuj Arora, Humbert Fiorino, Damien Pellier, Marc M' Etivier, Sylvie Pesty. A Review of Learning Planning Action Models. Knowledge Engineering Review, 2018, 33, 10.1017/S0269888918000188 . hal-02010536

\section{HAL Id: hal-02010536 \\ https://hal.science/hal-02010536}

Submitted on 7 Feb 2019

HAL is a multi-disciplinary open access archive for the deposit and dissemination of scientific research documents, whether they are published or not. The documents may come from teaching and research institutions in France or abroad, or from public or private research centers.
L'archive ouverte pluridisciplinaire HAL, est destinée au dépôt et à la diffusion de documents scientifiques de niveau recherche, publiés ou non, émanant des établissements d'enseignement et de recherche français ou étrangers, des laboratoires publics ou privés. 


\title{
A Review of Learning Planning Action Models
}

\author{
ANKUJ ARORA*
}

HUMBERT FIORINO*

DAMIEN PELLIER*

MARC MÉTIVIER**

SYLVIE PESTY*

${ }^{*}$ Univ. Grenoble Alpes, CNRS, Grenoble INP, LIG, 38000 Grenoble, France

E-mail: firstname.lastname@univ-grenoble-alpes.fr

**Paris Descartes University, LIPADE, 45 rue des Saints-Pŕes 75006 Paris, France

E-mail: marc.metivier@parisdescartes.fr

\begin{abstract}
Automated planning has been a continuous field of study since the $1960 \mathrm{~s}$, since the notion of accomplishing a task using an ordered set of actions resonates with almost every known activity domain. However, as we move from toy domains closer to the complex real world, these actions become increasingly difficult to codify. The reasons range from intense laborious effort, to intricacies so barely identifiable, that programming them is a challenge that presents itself much later in the process. In such domains, planners now leverage recent advancements in machine learning to learn action models i.e. blueprints of all the actions whose execution effectuates transitions in the system. This learning provides an opportunity for the evolution of the model towards a version more consistent and adapted to its environment, augmenting the probability of success of the plans. It is also a conscious effort to decrease laborious manual coding and increase quality. This paper presents a survey of the machine learning techniques applied for learning planning action models. It first describes the characteristics of learning systems. It then details the learning techniques that have been used in the literature during the past decades, and finally presents some open issues.
\end{abstract}

\section{Introduction}

Automated Planning (AP) is the branch of Artificial Intelligence pivoted on the formulation of a plan: a series of actions guiding the transition of the system from the initial state to the goal, accomplishing a required task in the process. Each action requires a certain number of preconditions to be fulfilled in order to be applied to a particular world state. Upon application, each action changes the world state with its induced effects. These actions can be represented by actions models: the blueprints of the domain-specific actions. AP came into light in the 1960s when research in the fields of operations research, theorem proving and studies into human problem solving started gathering steam, with the intent of solving problems that were being posed by the field of robotics among many others. The STRIPS (STanford Research Institute 
Problem Solver) system (Fikes \& Nilsson (1971)) was used to control and plan the movement of an autonomous robot called Shakey (Nilsson (1984)) as it pushed a series of boxes through a series of interconnected rooms. This is the perfect illustration of the application of AP to execute a task in a real life scenario. Planning, however, cannot be conducted in the absence of domain knowledge, which describes the actions that can be executed, serving as the information base to plan upon. This is where Knowledge Engineering (KE) finds its place in the domain of planning.

$\mathrm{KE}$ for $\mathrm{AP}$ is the process that deals with the acquisition, formulation, validation and maintenance of planning knowledge, where the essential output is the domain model (Jilani et al. (2014)). The main issue of current KE approaches for encoding domain models is that they require specific expertise which can only be furnished by a domain expert. This expert, in theory can, due to his limited knowledge of the real world domain, introduce some human-induced error in the encoding. In these situations, the success of the AP systems fully depends on the skills of the expert who defines the action model. This among others is the reason why AP, having enjoyed many feats and resounding successes in the aforementioned fields, it continues to suffer from its share of drawbacks.

Principal out of these drawbacks is the effort surrounding the creation of action models for complex domains. While it is possible to codify action models for simple domains, it remains laborious to do so for some complex real-world domains. Real world planning domain models are hard to develop, debug and maintain. As planners and applications become larger, the challenge of engineering planning domain models becomes more magnified (McCluskey et al. (2002)). Moreover, given the sheer difficulty in generating planning domain models, many users are not exploiting AP but easier though less-efficient approaches. It is worth pointing out that $\mathrm{KE}$ tools for encoding domain models are, usually, not very well known outside the planning community (Shah et al. (2013)).

With the intent of assisting domain experts, the planning community is developing systems to automatically acquire AP models (Jiménez et al. (2012)). Machine Learning (ML) seems to be a useful tool for these systems to automate the extraction, refinement, organization and exploitation of knowledge from plan traces or user preferences. ML is viewed as a potentially powerful means of making an agent (independent entity which perceives the world and performs actions accordingly) autonomous, while compensating for the expert's incomplete domain knowledge. The work done in ML goes hand in hand with the long history of domain independent planning, as the techniques developed as a part of this effort could be applied across a myriad of domains to accelerate planning. In most cases, the learning techniques introduced into planners were extensively customized to the specific architecture of the application domain. Therefore, any new and more powerful learning technique required programming effort to replace an existing one. These factors might explain why the planning community appears to have paid less attention to learning till the late 1990s. However in recent times, with the explosion in computing power and the influx of ML techniques into a myriad of computing fields, ML seems to have staged its comeback since the early 2000s.

This article is pivoted on ML techniques which allow the learning of the underlying planning model from a given set of traces consisting of plan executions. The employed learning techniques vary widely in terms of context of application, technique of application, adopted learning methodology and knowledge learned. The article begins with an introduction to the domain of AP to the reader (section 2), followed by an a description of certain key criteria that help in the characterization of the various learning systems (section 3). It then mentions interesting learning approaches in the literature (section 4), proceeds to highlighting some persisting open issues (section 5) with the discussed approaches, concluding with a roundup of the paper (section 6).

\section{Formulation and Representation}

Automated Planning (AP) is a means-end reasoning i.e. participating agents decide how to achieve a goal given a set of available and applicable actions. These agents interact with the environment 
by executing actions which change the state of the environment, gradually propelling it from its initial state towards the agents' desired goal. This transition can also be regarded as a solution to a planning problem the agent in question is looking to resolve. We begin with some definitions of fundamental concepts.

Definition 1 (Planning Problem). A planning problem is a triplet $\mathrm{P}=\left(S T S, s_{0}, g\right)$ composed of:

- the initial state $s_{0}$ of the world,

- the goal $g$, (set of propositions representing the goals to achieve), belonging to the set of goal states $S_{g}$, and

- the state transition system STS responsible for changing the world state by action application.

Definition 2 (STS). The STS in the previous definition is formally a 3-tuple $(S, A, \alpha)$ further composed of

- $\mathrm{S}=\left\{s_{1}, s_{2}, \ldots s_{n}\right\}$ as the set of states,

- $\mathrm{A}=\left\{a_{1}, a_{2}, \ldots a_{n}\right\}$ as the set of actions. Here each action $\mathrm{a} \in \mathrm{A}$ is defined in the form of the aggregation of three lists, namely (pre, add and del). These are the pre list (predicates whose satisfiability determines the applicability of the action), add list (predicates added to the current system state by the action application) and the del list (predicates deleted from the current system state upon action application), respectively. The listing of all the applicable actions in a domain is called the action model.

- $\delta:\left(S \times A^{*} \rightarrow S\right)$ is the state transition function (Ghallab et al. (2004)).

All the aforementioned elements in this section contribute to the formulation of a plan.

Definition 3 (Plan). A plan, given an initial state of the system, goal, and an action model, is a sequence of actions $\pi=\left[a_{1}, a_{2}, \ldots a_{n}\right]$ that drives the system from the initial state to the goal.

The transition from the initial state to the goal is driven by the previously mentioned transition function in Definition 1 (Ghallab et al. (2004)):

$$
\delta(s, \pi)= \begin{cases}s & \text { if }|\pi|=0 \\ \delta\left(\delta\left(s, a_{1}\right),\left[a_{2}, \ldots a_{k}\right]\right) & \text { if } \operatorname{precond}\left(a_{1}\right) \subseteq s\end{cases}
$$

This transition function, when applied to the set of the current state and the applicable action, produces the next state as:

$$
s_{i+1}=\delta\left(s_{i}, a\right)=\left(s_{i}-\operatorname{del}(a)\right) \cup \operatorname{add}(a)
$$

This transition function, when successively applied to the resulting intermediate states at each step, leads to the goal. Each such action sequence, complete with initial state and goal information (and occasionally intermediate state information), constitutes a trace. These traces can either be in the form of action sequences (as represented in figure 4) or action sequences interleaved with intermediate states. We explain the notion of traces in the form of a concrete example. AP defines a certain number of world domains, one of the most famous ones being Blocksworld (Winograd (1972)). It consists of a set of blocks resting on a table, the goal being to build one or more piles of blocks. However only one block may be moved at a time: either placed on top of another block or on the table. An example of action sequences in this domain is seen in the figure 4 . A snapshot of a state-action interleaved trace for the first two actions in figure 4 is given as : 
UNSTACK (blockD, blockC), (holding (blockD), not (armempty), clear ( blockC)), PUIDOWN (blockD)

Whilst good in theory, the creation of models in the case of complex domains is a non trivial task. While it is possible to codify, debug and maintain action models pertaining to simple toy domains, it remains laborious, unfeasible and sometimes impractical to do so for some complex real-world domains. The amount of effort needed to encode and maintain accurate action blueprints is significant. There is also no "one size fits all" strategy. Thus, we seek help from various ML techniques which allow learning of the underlying action model from traces produced as a result of plan execution. This model can ideally be re-injected into the planner for further planning purposes. The ML techniques to learn from these traces broadly fall into one of the following categories:

- Online: Learning occurs during the execution phase (Zimmermann \& Kambhampati (2003)). The system can start learning as soon as the generation phase is complete. This kind of learning never stops, thus allowing the continuous correction and improvement of an incorrect model and adaptation to changing environment characteristics. On the flip side, the cost of learning is added to the cost of planning, augmenting the time window in which execution occurs.

- Offline: One-shot learning exercise from traces. It allows a decoupling of the learning and planning phases, ensuring that the cost of learning is not added to that of planning. However, this one-time learning also means that in case of the injection of an ill defined domain, the planner may never be able to recover before the end of the planning and the beginning of the learning phase, thus staying blocked. Offline learning is the more popular learning mechanism.

Given the aforementioned information, our learning problem can be formulated as follows: given a set of plan traces $T$, to learn a domain model $m$ encompassing all the domain-applicable actions which best explains the observed plan traces (see figure 1).

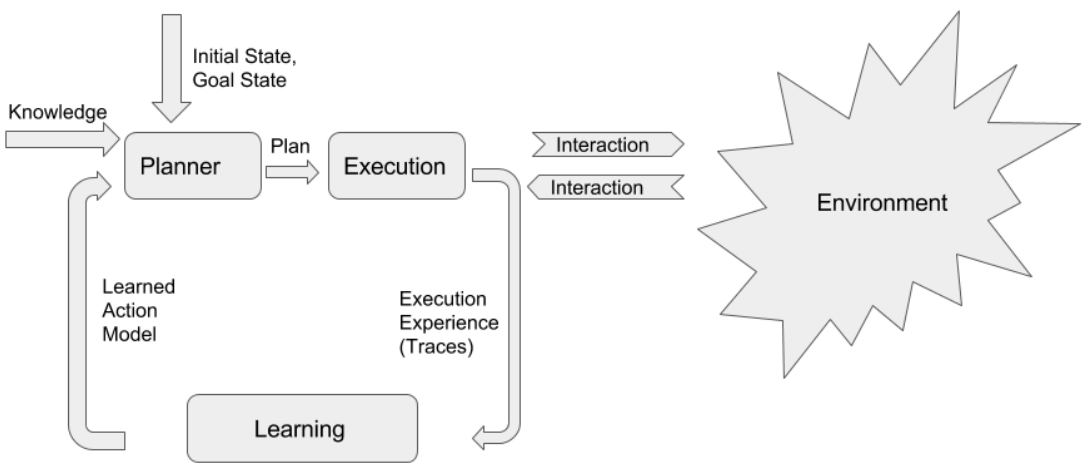

Figure 1: Formulation and Representation

We explain the aforementioned definitions in the form of a concrete example. A sample problem in the Blocksworld domain with its solution sequence is represented in the figures 2 and 3.

AP problems and domains are typically represented in a standard language called the Planning Domain Definition Language (PDDL). It has been the official language for the representation of the problems and solutions in all of the International Planning Competitions (IPC) which have been held from the year 1998 onwards. 


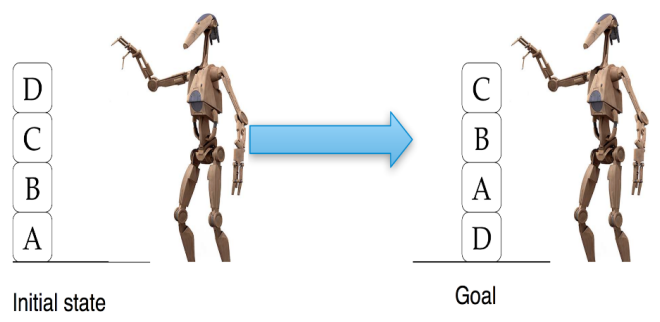

Figure 2: Problem definition

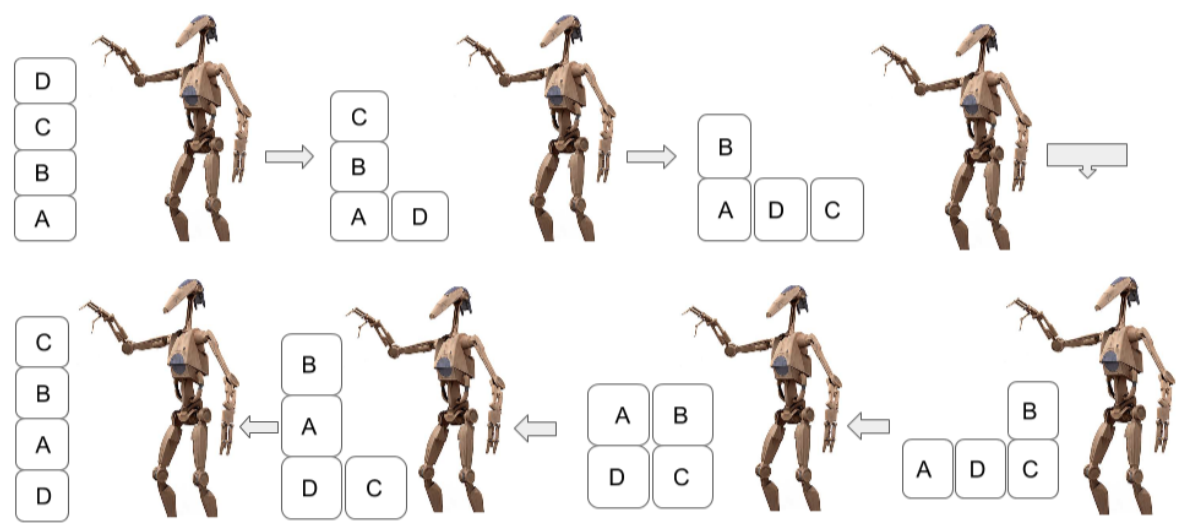

Figure 3: Solution steps

The PDDL representation of the actions and the sequence of steps leading to the final block configuration can be summarized in the figure 4. The left of the figure represents the sequence of actions of the Blocksworld domain, namely: pickup, putdown, stack, unstack . The right of the figure represents a magnified view into two of the four actions, namely stack and unstack. In the PDDL representation of these actions, the name of the action follows the : action tag. Similarly, the parameters constituting the action signature follow the : parameters tag. The preconditions and effects of the action are represented as a conjunction of predicates follow the :precondition (equivalent to the pre list in Definition 2) and :effect (equivalent to the add and del lists in Definition 2) tags respectively.

Choosing a good representation language which accurately models every action effect the system might encounter and no others is essential. Any extra modeling capacity is wasted and complicates learning, since the system will have to consider a larger number of potential models, and be more likely to overfit (Pasula et al. (2007)). 


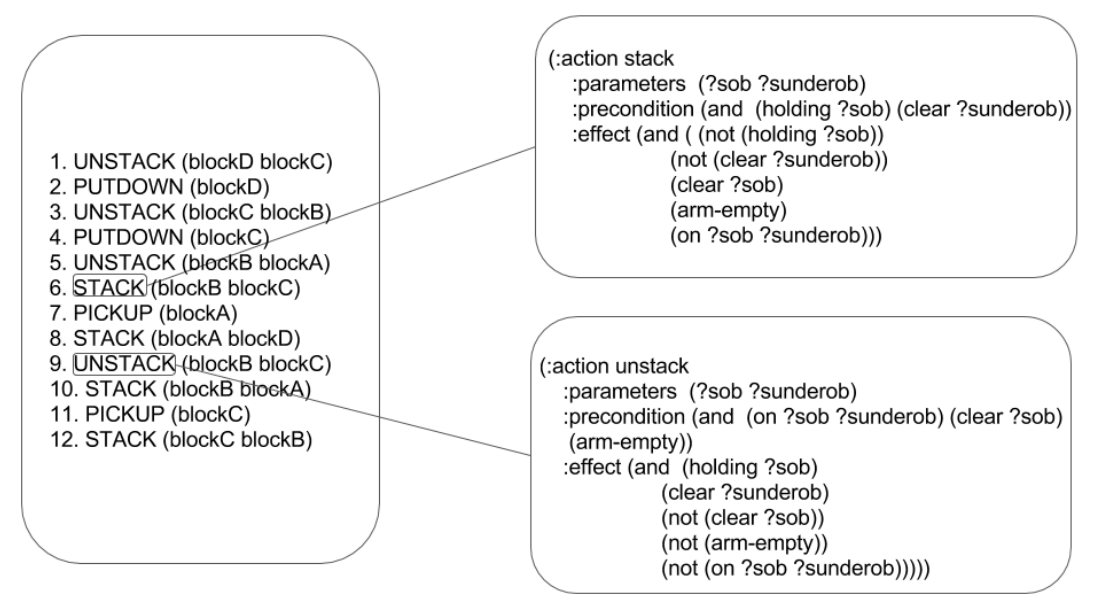

Figure 4: PDDL Representation Of the actions stack and unstack in the Blocksworld domain

\begin{tabular}{|l|l|l|}
\hline Language & Features & Limitations \\
\hline $\begin{array}{l}\text { PDDL } \\
\text { (McDermott } \text { et al. } \text { (1998)) })\end{array}$ & $\begin{array}{l}\text { (i) Machine-readable, standardized } \\
\text { syntax for representing STRIPS } \\
\text { and other languages. } \\
\text { (ii) Has types, constants, predicates } \\
\text { and actions }\end{array}$ & $\begin{array}{l}\text { Goal cannot be } \\
\text { included into } \\
\text { action models }\end{array}$ \\
\hline $\begin{array}{l}\text { PPDDL } \\
\text { (Younes } \text { et al. } \text { (2005)) }\end{array}$ & $\begin{array}{l}\text { as the standard high } \\
\text { level planning language } \\
\text { for probabilistic planners } \\
\text { (ii) Supports actions with } \\
\text { probabilistic effects }\end{array}$ & $\begin{array}{l}\text { Goal cannot be } \\
\text { included into } \\
\text { action models }\end{array}$ \\
\hline $\begin{array}{l}\text { STRIPS } \\
\text { (Fikes \& Nilsson (1971)) }\end{array}$ & $\begin{array}{l}\text { Sublanguage of PDDL } \\
\text { OCL }\end{array}$ & $\begin{array}{l}\text { (i) Accounts for } \\
\text { deterministic not } \\
\text { probabilistic actions } \\
\text { (Mi) Requires exponential } \\
\text { number of samples to learn }\end{array}$ \\
\hline et al. $(2002))$ & $\begin{array}{l}\text { High level language with } \\
\text { representation centered } \\
\text { around objects instead } \\
\text { of states }\end{array}$ & $\begin{array}{l}\text { Cannot be input } \\
\text { into standard } \\
\text { planners }\end{array}$ \\
\hline $\begin{array}{l}\text { RDDL } \\
\text { STRIPS + functional terms, } \\
\text { leading to higher expressiveness }\end{array}$ & $\begin{array}{l}\text { STRIPS operators augmented with } \\
\text { quantifiers and conditional effects, } \\
\text { resulting in situational } \\
\text { calculus-like expressiveness }\end{array}$ & $\begin{array}{l}\text { Does not cater } \\
\text { to non-determinism }\end{array}$ \\
\hline $\begin{array}{l}\text { ADL } \\
\text { (Pednault (1989)) }\end{array}$ & $\begin{array}{l}\text { Computational efficiency } \\
\text { proportional to } \\
\text { knowledge of initial state }\end{array}$ \\
\hline
\end{tabular}

Table 1: Representation Languages in AP (PDDL = Planning Domain Definition Language, PPDDL $=$ Probabilistic PDDL, OCL $=$ Object Centered Language, $\mathrm{RDDL}=$ Relational Dynamic influence Diagram Language, $\mathrm{ADL}=$ Action Description Language)

Some languages in the domain of $\mathrm{AP}$ and their features are summarized in the Table 1. We return to our example of the Blocksworld domain, and refer to the action "pick-up" 
which represents the picking up of a block from the table or from another block. A syntactic representation of the action "pick-up" of the Blocksworld domain in four prominent languages (described in Table 1) can be found in Table 2.

\begin{tabular}{|c|c|c|}
\hline Language & Domain & Action Definition \\
\hline PDDL & \multirow{2}{*}{$\begin{array}{l}\text { \%Definition of the blocksworld domain } \\
\text { (define (domain BLOCKSWORLD) } \\
\text { \%Declaration of used packages (strips, typing) } \\
\text { (:requirements :strips :typing) } \\
\text { \%Declaration of constant objects } \\
\text { (:types block) } \\
\text { \% Declaration of symbolic facts } \\
\text { (:predicates (on ?x - block ?y - block) } \\
\text { (ontable ?x - block) (clear ?x - block) } \\
\text { (handempty) (holding ?x - block)) }\end{array}$} & $\begin{array}{l}\text { \% Action definition } \\
\text { (:action pick-up } \\
\text { :parameters (?x - block) } \\
\% \text { Preconditions for action applicability } \\
\text { :precondition (and (clear ?x) (ontable ?x) } \\
\text { (handempty)) } \\
\text { \% Transitions for objects changed by the action } \\
\text { :effect(and (not (ontable ?x)) (not (clear ?x)) } \\
\text { (not (handempty)) (holding ?x))) }\end{array}$ \\
\hline PPDDL & & $\begin{array}{l}\text { \% Action definition } \\
\text { (:action pick-up } \\
\text { :parameters (?x - block) } \\
\% \text { Prevailing conditions for operator applicability } \\
\text { :precondition (and (clear ?x) (ontable ?x)) } \\
\text { \% Transitions for objects changed by the action } \\
\text { :effect (and (when (not (handempty)) } \\
\text { (probabilistic 0.00) (and (clear ?x) (ontable ?x)) } \\
\text { (when (handempty) } \\
\text { (probabilistic 1.00) (and (not (ontable ?x)) } \\
\text { (not (clear ?x)) (not (handempty)) (holding ?x))) }\end{array}$ \\
\hline STRIPS & $\begin{array}{l}\text { \%Definition of the blocksworld domain } \\
\text { (define (domain BLOCKSWORLD) } \\
\text { \%Declaration of used packages (strips) } \\
\text { (:requirements :strips) } \\
\text { \% Declaration of symbolic facts } \\
\text { (:predicates (on ?x ?y) } \\
\text { (ontable ?x) (clear ?x) } \\
\text { (handempty) (holding ?x)) } \\
\% \text { Action definition } \\
\text { (:action pick-up } \\
\text { :parameters (?x) } \\
\text { \% Preconditions for operator applicability } \\
\text { :precondition (and (clear ?x) (ontable ?x) } \\
\text { (handempty)) } \\
\text { \% Transitions for objects changed by action } \\
\text { :effect(and (not (ontable ?x)) (not (clear ?x)) } \\
\text { (not (handempty)) (holding ?x))) }\end{array}$ & $\begin{array}{l}\text { Action representation in STRIPS } \\
\text { :action (pick-up (b) } \\
P R E C O N D: \text { clear }(b) \wedge \text { ontable }(b) \wedge \text { handempty } \\
E F F E C T: \neg \text { ontable }(b) \wedge \neg \text { clear }(b) \\
\wedge \neg \text { handempty } \wedge \text { holding }(x)\end{array}$ \\
\hline
\end{tabular}




\begin{tabular}{|c|c|c|}
\hline OCL & $\begin{array}{l}\text { \% objects(Type and instances of each object) } \\
\text { objects(block, [block1]) } \\
\text { objects(gripper, [tom]) } \\
\% \text { All predicates } \\
\text { predicates([on_block(block,block), } \\
\text { on_table(block), clear(block), } \\
\text { gripped(block,gripper), busy(gripper), } \\
\text { free(gripper)]) }\end{array}$ & $\begin{array}{l}\text { \%Substrate Class (sc): "typical situations" that an } \\
\text { object of a particular sort may exist in as result of } \\
\text { planning process } \\
\text { operator( grip_from_table }(\mathrm{B}, \mathrm{G}) \\
\% \text { prevail (preconditions for operator } \\
\text { applicability) } \\
{[]} \\
\% \text { necessary (transitions for objects } \\
\text { changed by action) } \\
{\left[\mathrm{sc}\left(\text { block, } \mathrm{B},\left[\mathrm{on} \_ \text {table }(\mathrm{B}), \text { clear }(\mathrm{B})\right]\right.\right.} \\
=>[\text { gripped }(\mathrm{B}, \mathrm{G})]), \\
\mathrm{sc}(\text { gripper, },[\text { [ree }(\mathrm{G})]=>[\text { busy }(\mathrm{G})])] \\
\% \text { conditional }(\text { optional conditions) } \\
{[]} \\
\text { ) }\end{array}$ \\
\hline
\end{tabular}

Table 2: Representation of action 'pick-up' of the Blocksworld domain in various languages

\section{Characteristics of Learning Systems}

During the course of this bibliographical review, we have been able to identify certain key criteria which help us characterize the works in this paper. These criteria are described in the following subsections. These criteria also serve as a guideline for creating a "cheat sheet" with the intent of helping researchers starting out in the domain to choose a specific learning technique based on the characteristics defined in this section. This "cheat sheet" (see figure 5) is not exhaustive, and conceptualized bearing in mind the methodology adopted by the paper and the learning techniques discussed in section 4 . The branch in this figure dedicated to the learning of heuristics is not detailed as it is beyond the scope of this paper. The characteristics are detailed in the subsections below.

\subsection{Representation Mechanism}

This section tries to classify approaches based on the viewpoint the learning system is observed from. The learning system can be seen as:

- Object-centered representation: Each object in the domain manipulated by an action is represented by a single parameterized state machine. The action model is generally represented in OCL (Object Centered Language).

- System-centered representation: A plan is viewed as an interleaved sequence of states and actions representing the transition of the system from the initial state to the goal. The action model in this case is generally represented in the form of PDDL and its sub-languages or variants.

\subsection{Inputs To The System}

The inputs to the learning system as well as some other characteristics (quality, quantity etc.) go a long way in determining the output and its quality. The inputs to the system may include: the model, the background knowledge and the traces; all three in varying capacities. 


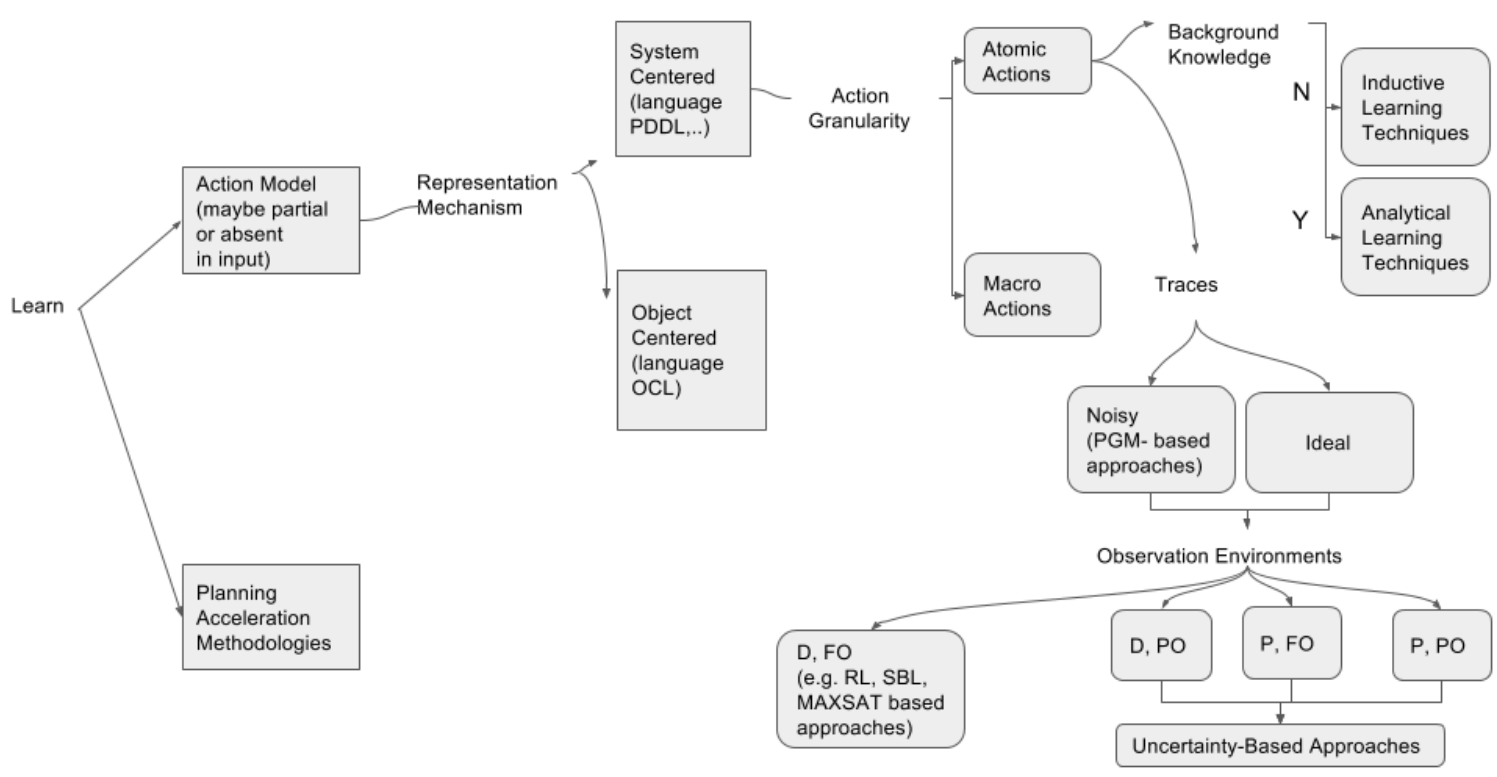

Figure 5: Guideline for choosing appropriate learning approach based on input requirements (OCL $=$ Object Centered Language, $\mathrm{D}=$ Deterministic, $\mathrm{P}=$ Probabilistic, $\mathrm{FO}=$ Fully Observable, $\mathrm{PO}=$ Partially Observable, MAXSAT=Maximum Satisfiability, PGM=Probabilistic Graphical Model, RL $=$ Reinforcement Learning, SBL $=$ Surprise Based Learning)

\subsubsection{Model}

Before the learning phase begins, the action model may exist in one of the following capacities:

- No Model: This refers to the fact that no information on the actions that constitute the model is available in the beginning, and the entire model must be learnt from scratch.

- Partial Model: Some elements of the model are available to the learner in the beginning, and the model is enriched with more knowledge at the end of the learning phase.

\subsubsection{Background Knowledge}

Background knowledge (BK) is mostly required only in the absence of complete and correct domain knowledge. The availability of this BK is inversely proportional to the planning effort required. For example in the blocksworld domain, "only one block can be on top of some other block" or "a block being held means that the robotic arm is not empty" is an illustration of BK. To compensate for the absence of domain knowledge, this BK may also comprise of object type definitions as well as predicate and intermediate state information (Jilani et al. (2014)).

In most cases, it restricts the possible action choices at every system state, thus speeding up planning. This BK, however, may also be difficult to acquire. Depending on the presence or absence of background knowledge, the associated learning techniques may either be classified as analytical or inductive respectively. These techniques are discussed in detail in section 4.1.

\subsubsection{Traces}

Traces are the residue or the proof of the execution of a series of actions in a domain. They represent a summary of the executed action and the resulting state of the world. These plan execution traces may be classified into pure or adulterated as follows: 
- Noisy: The traces can be adulterated because of sensor miscalibration or faulty annotation by a domain expert. For example, AMAN (Action-Model Acquisition from Noisy plan traces) (Zhuo et al. (2013)) falls into this category.

- Ideal: There is no discrepancy between the ideal action and the recorded action. For example, OBSERVER (Wang (1996)).

\subsection{System Outputs}

\subsubsection{Action Granularity}

Irrespective of whether the trace is represented in form of action sequences or state-action interleavings, the actions can be classified as atomic or grouped. Atomic actions are unitary actions applied to a system state to effectuate a transition. Macro actions, on the other hand, are a group of actions applied at a time point like a single action, representing high level tasks while encompassing low level details (Newton et al. (2007, 2008, 2010)). From a broader perspective, macros are like procedures in the programming realm. They are promising because they are capable of aggregating several steps in the state space, and providing extended search space visibility to the planner. Some merits of using macros include:

- Macros generated from plans of smaller problems are scalable and can be evaluated against larger problems.

- Macros allow planning of several steps at a time and allow for re-usability in plan snippets. Knowledge acquired from macros can be integrated into the planner or encoded into the domain (Newton et al. $(2007,2008,2010))$.

Some limitations are as follows:

- They increase the processing time of the planner, adding more branches in the search tree at every point in time. However, the problem is significantly reduced by search tree pruning used by many recent planners (Newton et al. (2007)).

- Macros often result in longer plans than when no macro is used.

\subsubsection{State Observability and Action Effects}

The observability of the current state of the system after the action execution may either be perfectly certain or flawed because of faulty sensor calibration. In cases of faulty calibration, the state of the system is only partially observable at run time, thus observations return a set of states (called as "belief states") instead of a single state (Ghallab et al. (2004)).

Similarly, actions are not necessarily deterministic (single outcome on execution) but may be stochastic, that is: capable of producing multiple outcomes, each of them equipped with a different execution probability.

Keeping these variations of action effects and state observability in mind, we define four categories of implementations:

- Deterministic effects, Full state observability: For example, the EXPO (Gil (1992)) system.

- Deterministic Effects, Partial State Observability: In this family, the system may be in one of a set of "belief states" after the execution of each action (Ghallab et al. (2004)).

For example, the ARPlaces system (Stulp et al. (2012)).

- Probabilistic effects, Full State Observability: for example (Jiménez et al. (2012), Deshpande et al. (2007)).

- Probabilistic Effects, Partial State Observability: Barring a few initial works in this area (Yoon \& Kambhampati (2007)), this classification remains as the most understudied one till date. 


\subsection{Learnt Model Granularity}

Learning systems can be also be characterized according to the sheer amount of detail represented in the output representation of the learnt model. This can range from a bare-bones STRIPS model produced in the output (Zhuo \& Kambhampati (2013)), to PDDL models with quantifiers and logical implications (Zhuo et al. (2010)), to PDDL models with static predicates (predicates existing implicitly in operator preconditions and never appearing in plans e.g. Jilani et al. (2015)). Granularity is used a criteria to classify the works summarized in the Table 3 based on the richness of the information learnt based on the amount of input provided.

\section{Type of Learning}

This section sheds light on some classical learning techniques which have been around and in use for learning action models, as well as newer and interesting techniques which have emerged with recent advancements and the broadening spectrum of ML techniques. The algorithms described in this paper are further summarized in the form of Table 3. These tables provide a bird's eye view of the key characteristics of each algorithm in terms of input provided, output produced, application environment etc. These tables are described with the intent of serving someone who is new to the domain and is looking to situate their problem in the context of a previously done work.

\subsection{Learning Techniques based on availability of background knowledge}

As discussed in section 3.2.2, the learning techniques based on the absence or presence of background knowledge are classified into inductive and analytical learning techniques respectively.

\subsubsection{Inductive Learning}

The learning system is fed with a hypothesis space $H$ and a set of traces $T$. The desired output is a hypothesis from the space $H$ that is consistent with these traces (Zimmermann \& Kambhampati (2003)). Inductive techniques are useful because they can identify patterns and generalize over many examples in the absence of a domain model. One prominent inductive learning technique is that of regression tree learning. Regression trees are capable of predicting continuous variables and modeling noise in the data. In comparison to a decision tree which predicts along a category (i.e. class), a regression tree performs value prediction along the dependent dimension for all observations (Balac et al. (2000)).

The PELA (Planning, Execution and Learning Architecture) system (Jiménez et al. (2008)) performs the three functions suggested in its name to generate probabilistic rules from plan executions and compile them to refine its planning model. This is done by performing multiclass classification, which consists of using the TDIDT (Top-Down Induction of Decision Trees) (Quinlan (1986)) algorithm to find the smallest decision tree that fits a given data set. A decision tree is built from examples from which probabilities are captured to refine its initial model.

In ERA (Exploration, Regression tree induction and Action models) (Balac et al. (2000)), a robot learns action models for its navigation under various terrain conditions. With each exploration step, the robot records the executed action, the current terrain conditions and positions before and after the action execution. When exploration is finished, the data is divided into groups based on action type and a regression tree induction algorithm is applied to the data for each action type. This algorithm then builds a regression tree representing the model and expected outcome of that particular action.

LOCM (Cresswell et al. $(2009,2013)$ ) uses an object-centered representation and learns a set of parameterized Finite State Machines (FSM), where each FSM represents pre and post-conditions of the domain operators (Jilani et al. (2014)). A planning domain consists of sets (called sorts) of object instances, where each object behaves in the same way as any other object in its sort. LOCM first collects the set of all transitions in the example sequences. It then derives a set of 
state machines, each of which can be identified with a sort. It then performs inductive learning and identifies state parameters, providing correlations between the action parameters and state parameters occurring in the start/end states of transitions (Cresswell et al. (2009, 2013)). The limitation of LOCM is that it provides a single state machine to represent each object, which is overcome by LOCM2 (Cresswell \& Gregory (2011)).

LOCM2 (Cresswell \& Gregory (2011)) is generalized and heuristic in nature to allow multiple state machines to represent a single object. This extends the coverage of domains for which a domain model can be learned. Unlike LOCM, LOCM2 constructs many possible solutions to the same problem. The heuristic is used to select a single solution from these possible solutions. This means that the learned models produce plans which are shorter than optimal solutions. Note that both systems gather only dynamic properties of a planning domain and not the static ones. This is a drawback as many systems use static predicates to contain the set of possible actions. This limitation is addressed by the NLOCM (Numeric LOCM) system.

NLOCM (Gregory \& Lindsay (2016)) extends classical planning ${ }^{1}$ to numerical planning i.e. learns action costs in planning domains from state transition sequences using a constraint programming approach. It uses the LOCM2 and LOP systems as pre-processing steps. While the LOCM and LOCM2 algorithms only learn the dynamic aspects of the domain, the LOP system (Gregory P. \& Cresswell (2015)) learns static relations. NLOCM extends the FSM of LOCM to an automata with numeric weights on the important features. A set of templates is defined for each operator which contributes to the action cost of that operator in the domain. The algorithm successively solves more and more complex template sets until a satisfying assignment which minimizes the complexity cost is found. These templates are used to find the valid cost models.

Opmaker (McCluskey et al. (2002)) is a mixed initiative tool to induce operators from action sequences, domain knowledge and user interaction. These operators are parameterized and hierarchical in nature. OCL is used to model the domain. For each action in the training solution sequence, it asks the user to input, if needed, the target state that each object would occupy after the action execution, thus creating its operator schema step-by-step. It is implemented inside a graphic tool called GIPO (Graphical Interface for Planning with Objects), which facilitates user interaction, domain modeling and operator induction (McCluskey et al. (2002), Jilani et al. (2014)).

Opmaker2 (McCluskey et al. (2009)) is an improvement over Opmaker such that it eliminates the dependency on the user for intermediate state information. After Opmaker2 automatically infers this intermediate state information, it proceeds in the same fashion as Opmaker and induces the same operators. The main advantage of Opmaker2 is that it computes its own intermediate states using a combination of heuristics and inference from the partial domain model (PDM), example sequences and solutions. In Opmaker2, the DetermineStates procedure performs this function by tracking the changing states of each object referred to in the training example, taking advantage of the static and other information in the PDM. The output from DetermineStates is, for each object, a map associating each object to a unique state value at each point in the training sequence. Once the map has been generated, the techniques of the original Opmaker algorithm are used to create an operator schema.

In Martínez et al. (2016), the authors learn a probabilistic model including exogenous effects (predicates not related to any action execution) from a set of state transitions. They use the LFIT (Learning From Interpretation Transitions) framework (Inoue et al. (2014)) which uses ILP to induce a set of propositional rules that orchestrate the given input transitions. This is done by first transforming grounded to relational transitions, then relational transitions to probabilistic

\footnotetext{
${ }^{1}$ Branch of planning in which predicates are propositional: they do not change unless acted upon by the planning agent. Moreover, all relevant attributes can be observed at any time, the impact of action execution on the environment is known and deterministic, the effects of action execution occur instantly and so on (Zimmermann \& Kambhampati (2003)).
} 
rules. Planning operators can be reconstructed from probabilistic rules. A score function evaluates the operators, and a heuristic search algorithm selects the set of operators that maximize the score.

\subsubsection{Analytical Learning}

The learning system has at its disposal the same hypothesis space $H$ and traces as in the case of inductive learning but with an additional input: BK that can explain traces. The desired output is a hypothesis from the space $H$ that is consistent with both the traces and BK. Analytic learning relies on BK to analyze a given trace and identify its relevant features. Any system which relies on domain knowledge, predicate information or intermediate state information can be classified under this category. More details about analytic learning techniques can be found in (Zimmermann \& Kambhampati (2003)).

\subsection{Genetic and Evolutionary algorithm based approaches}

A series of approaches which use evolutionary algorithms to learn macro actions in the absence of any kind of additional knowledge may be classified under this category. An approach by (Newton et al. (2007)) learns macro actions given a domain and action sequences. It is generic in the sense that it does not require structural knowledge about the domain or the planner, but learns from plans generated for random planners and domains using a genetic algorithm .

Another approach by the same author (Newton \& Levine (2010)) learns macros as well as macro-sets (collection of macros which collectively perform well). In this work, once a macro is learned, it is tinkered with by adding, deleting and modifying existing and new actions in a bid to learn how the new macro performs. In the first phase, only individual macros are learned using actions from generalized plans produced by solving input problems. In the second phase, macro-sets are learned using the macro pool obtained in the first phase, including only those that have fitness values greater than a certain minimum level.

In a third approach by the same author (Newton et al. (2008)), non-observable macros i.e. macros that are not observable from traces are learned. This is done with the intent of constructing a comprehensive macro-library for future reuse. Learning is based on an evolutionary algorithm. Evolutionary algorithms repeatedly generate new macros (in every epoch) from current individuals by using given genetic operators: only the individuals with the best fitness values survive through successive epochs. The fitness function is based on three measures: Cover, Score, Point. Cover evaluates the percentage of problems solved when the macro is used. Score measures the time gained or lost over all the problems solved with the augmented domain compared to when they are solved using the original domain. Point measures the percentage of problems solved with the augmented domain which take less or equal time compared to when they are solved using the original domain.

\subsection{Reinforcement Learning}

Reinforcement learning (RL) is a special case of inductive learning, and more specifically characterizes a learning problem instead of a learning technique (Sutton \& Barto (1998)). An RL problem is composed of three principal elements: (i) the goal an agent must achieve, (ii) a stochastic environment, and (iii) actions an agent takes to modify the state of the environment (Sutton \& Barto (1998)). RL takes place during the process of plan building. Through trialand-error online traversal of states, an optimal policy (mapping from perceived states of the environment to actions to be taken when in those states) is found for achieving the agent's goals (Zimmermann \& Kambhampati (2003)). The strength of RL lies in its ability to handle dynamic and stochastic environments where the domain model is either nonexistent or incomplete. The benefits of an RL system are two-fold: on one hand, it improves plan quality (the system 
progressively moves towards finding an optimal policy) and, on the other hand, it can learn the domain model. One of the major drawbacks of RL is that of generalization: in its bid to achieve domain-specific goals, it cannot gather general knowledge of the system dynamics, leading to a problem of generalization. Despite its drawbacks, RL played a major role in the success of real life systems such as the recent AlphaGo (Silver et al. (2016)). AlphaGo used policy gradient RL (policy represented by own function approximator) to optimize a deep convolutional neural network by simulating games of the network playing against previous versions of itself and rewarding itself for taking moves that gave it wins.

The LOPE (Learning by Observation in Planning Environments) (García-Martínez \& Borrajo (2000)) system learned planning operators by observing the consequences of the execution of planned actions in the environment. In the beginning, the system is a blank slate and has no knowledge, it perceives the initial situation, and selects a random action to execute in the environment. It then loops by (1) executing an action, (2) perceiving the resulting situation of the action execution and its utility, (3) learning a model from this perception and (4) planning for further interaction with the environment. A global reinforcement mechanism either rewards or punishes operators based on their success in predicting the environment behavior. This is done by a virtual generalized $\mathrm{Q}$ table (associates each $(s, a)$ pair with an estimate of $Q(s, a)$, where $s$ and $a$ are state and action respectively, and $Q(s, a)$ is the expected reward for taking action $a$ in state $s)$.

In an approach by (Safaei \& Ghassem-Sani (2007)), probabilistic planning operators are learned in an incremental fashion. It has an integrated learning-planning system consisting of (i) an observation module for operator learning, (ii) an acting module for planning, and (iii) a control center for generating goals to further test the algorithm. This control center also dynamically assigns the reward function.

\subsubsection{Relational Reinforcement Learning}

Relational reinforcement learning is the combination between reinforcement learning and inductive logic programming (Dz̃eroski et al. (2001)). Given a (i) possible state set $S$, (ii) possible action set $A$, (iii) state transition function $\delta: S \times A \rightarrow S$, (iv) reward function $r: S \times A \rightarrow S$, (v) background knowledge and (vi) declarative bias for learning policy representations; the task is finding a policy $\pi$ for selecting actions $\pi: S \rightarrow A$ that maximizes the expected reward. RRL employs the Q-learning method (learns policies which are represented as value-assigned stateaction pairs) using a relational regression tree. Due to the use of a more expressive relational language, RRL can be applied to a wider array of problems. It also allows the reuse of results of previous learning phases and their application to novel and more challenging situations.

MARLIE (Model-Assisted Reinforcement Learning in Expressive languages) is the first RRL system that learns a transition and reward function online (Croonenborghs et al. (2007)). MARLIE builds probabilistic models accounting for the uncertainty of world dynamics. It uses the TG algorithm (Driessens et al. (2001)) to learn relational decision trees. The decision trees are not restructured on the appearance of new examples. However their fully relational aspect allows them to be applicable to a wider range of problems.

IRALe (Incremental Relational Action Learning algorithm) (Rodrigues et al. (2011)) produces a STRIPS-like (see Table 1) action model consisting of a set of rules by combining RRL and active learning. At first, the action model is an empty rule-set. The interactions between the agent and the environment produce examples. In the event of a counter example (an example that contradicts the model), the example has to be revised by modifying or adding one or more rules. This contradiction is determined by matching operations performed between the rules and examples, and each identified counter-example leads to either a specialization or a generalization. The agent also performs active learning, that is, in every state it chooses an action that it expects to lead to a generalization of the model. IRALe is mainly bottom-up, and new opportunities 
for generalizing the model will decrease the number of examples required to converge to the correct model. It uses a formalism called Extended Deterministic STRIPS (EDS), which is more expressive than traditional STRIPS.

\section{$4.4 \quad$ Uncertainty-based techniques}

\subsubsection{Markov Logic Networks}

A Markov logic network (Richardson \& Domingos (2006)) is a combination of first order logic and probabilistic graphical models. It is a first-order knowledge base consisting of weighted formulas, and can be equated to a template used to construct Markov networks. They have numerous advantages. From the probability point of view, MLNs provide a language scalable enough to specify very large Markov networks, and the ability to inject a comprehensive range of domain knowledge into them. From the first-order logic point of view, MLNs are equipped with the ability to soundly handle imperfect knowledge and uncertainty. For example, the LAMP (Learning Action Models from Plan traces) system (Zhuo et al. (2010)) learns action models with quantifiers and logical implications using MLNs. Firstly, the input plan traces (state-action inter leavings) are encoded into propositional formulas (conjunction of ground literals) to store into a database as a collection of facts. These formulas are very close to PDDL (refer to Table 1) in terms of representation. Secondly, candidate formulas are generated according to the predicate lists and domain constraints. Thirdly, a Markov Logic Network (MLN) uses the formulas generated in the above two steps to learn the corresponding weights of formulas and select the most likely subset from the candidate formula set. Finally, this subset is transformed into the final action models (Zhuo et al. (2010)).

\subsubsection{Noisy Trace Treatment Approaches}

As explained in section 3.2.3, sensor miscalibration may cause introduce noise giving birth to uncertainty. For example, AMAN (Action-Model Acquisition from Noisy plan traces) handles noise (Zhuo et al. (2013)) by finding a domain model that best explains the observed noisy plan traces using a probabilistic graphical model based approach. The only input to the learning phase are traces in the form of noisy action sequences. A probabilistic graphical model (PGM, graphical representation of dependence among random variables) is used to capture the relationship between the current state, correct action, observed action and the domain model. First, a set of all possible candidate models is generated. Then the observed noisy plan traces are used to predict the correct plan traces based on the PGM. Then, the correct plan traces are executed to calculate the reward of the predicted correct plan traces according to a predefined reward function. This reward function output is used to update the weights of the candidate models. The model with the highest weight is rendered as the model being searched for (Jilani et al. (2014)).

In a series of works by (Pasula et al. (2004, 2007), Zettlemoyer et al. (2005)), the goal is to learn action models in noisy and stochastic domains. This is done by: (i) Allowing rules to refer to objects not mentioned in the signature of the action. (ii) Relaxing the frame assumption (unspecified atoms in the operator's effects remain unchanged) and allowing the existence of "noise-induced" changes in the world. (iii) Extending the language: introducing newer and more complex like existential quantification, universal quantification, counting and transitive closure. It allows to learn probabilistic rules which are affected by a factor of noise and include deictic references, thus are called Noisy Deictic Rules (NDR). Deictic references are used to identify objects, even those which are not directly affected by the mentioned predicates. The works conclude that the addition of noise and deictic references increases the quality of the learned rules. 


\subsection{Surprise Based Learning (SBL)}

In a learning system, a surprise is produced if the latest prediction and the latest observation are considerably different (Ranasinghe \& Shen (2008)). Once an action is performed, the world state is sensed by a perceptor module which extracts sensor information. If the algorithm had made a prediction, it is verified by the surprise analyzer. If the prediction turns out incorrect, the model modifier adjusts the world model accordingly. Based on the updated model, the action selector will perform the next action so as to repeat the learning cycle. This notion of isolating faults is ideologically equivalent to the model based diagnosis $(M B D)$ approaches to explain faults presented in (De Jonge et al. (2009), Kalech (2012), Micalizio \& Torasso (2014)).

A series of approaches based on SBL have used Goal Driven Autonomy (GDA). GDA (Weber et al. (2012)) is a conceptual model for creating an autonomous agent that (i) monitors expectations during plan execution (ii) detects the occurrence of discrepancies, (iii) builds explanations and new goals to pursue in the case of failures. In order to identify when planning failures occur, a GDA agent requires the planning component to generate an expectation of world state after executing each action in the execution environment. The GDA model thus provides a framework for creating agents capable of responding to unforeseen failures during plan execution in complex environments.

FOOLMETWICE (Molineaux \& Aha (2014)) is a goal-oriented algorithm which learns from surprises. It tries to find inaccuracies in the environment model by attempting to explain all received observations. When a consistent explanation cannot be found, it infers that some unknown event happened that is not represented in the model. It then creates a model of the preconditions of these events by generalizing over the states that trigger them.

LIVE (Shen (1993)) is a discrimination-based learning method that creates general rules by noticing the changes in the environment, then specifying the rules by explaining their failures in prediction. When a rule executes and the predicted outcome does not match the observation, LIVE explores and creates new sibling rules. These rules are usually created by comparing the difference between a successful previous execution and the current failed one. New rules can accordingly be made more specific/generic.

LGDA (Learning GDA) (Jaidee et al. (2011)) is a GDA algorithm that uses case-based reasoning to map state-action pairs to a distribution over expected states, and map goal-discrepancy pairs to a value distribution over discrepancy-resolution goals. It also uses reinforcement learning to learn the expected values of the goals. It models goal formulation as an RL problem in which the value of a goal is estimated based on the expected future reward obtained on achieving it.

OBSERVER (Wang (1996)) is an integrated learning, planning and execution system which incrementally learns planning operators. The learning module learns operator preconditions and effects by observing experts executing sample problems, then refining the operators collected from these observations. The operator preconditions are learned by creating and updating both the most specific and most general representations of the preconditions based on trace executions. Operator effects are learned by generalizing the delta-state (the difference between pre-state and post-state) from multiple observations. This learning mechanism adopted by OBSERVER can also be seen in many following works (Walsh \& Littman (2008), Stern \& Brendan (2017)).

The EXPO (Gil (1992)) system refines incomplete planning operators by a method called the ORM (operator refinement method). EXPO does this by generating plans and monitoring their execution to detect the difference between the predicted and observed state. EXPO then constructs a set of specific hypotheses to fix the detected differences. After heuristic filtration, each hypothesis is experimentally tested and a plan is constructed to achieve the required situation (Jiménez et al. (2012)).

The ARTUE (Autonomous Response To Unexpected Events) system (Molineaux et al. (2010)) dynamically reasons about the goals to pursue when encountered with unexpected environmental situations. It consists of four principal components. A Hierarchical Task Network (HTN) planner reasons about exogenous events. A goal generation component reasons about and generates new 
goals. An explanation component reasons about hidden information in the environment. Finally, a goal management component manages and communicates the goals to the planner. ARTUE handles unexpected environmental changes by first explaining those changes, then generating new goals incorporating the explained knowledge about hidden environmental aspects.

\subsection{Transfer Learning}

Transfer learning is the technique which, given a source task and target task, aims to extract the knowledge from the source task and apply it to the target task in a situation where the latter has fewer training data (Pan \& Yang (2010)). Most machine learning methods work well only under a common assumption: the training and test data are drawn from the same probability distribution. However, when the distribution changes, most models need to be reconstructed, trained and tested from scratch using data generated from the new probability distribution. In many real world applications, it is expensive, if not impossible, to regenerate the needed training data for model reconstruction. In order to reduce the effort to regenerate the training data, the possibility of knowledge transfer between domains would be ideal. Transfer learning (Pan \& Yang (2010)) allows the domains, tasks, and probability distributions used in training and testing to be different. For example, we may find that learning to recognize apples might help to recognize oranges. Some characteristics of transfer learning systems include: the type of knowledge to be transfered, situation of transfer and technique of transfer. As far as type of knowledge goes, some knowledge is specific to individual domains, while other may be common between different domains such that it may help improve performance for the target domain. After discovering which knowledge can be transferred, learning algorithms need to be developed to perform the knowledge transfer. The situation of transfer is an important characteristic as while it is important to know the situation in which a transfer must occur, it is equally important to know the situations in which transfer must not occur. In some cases, when the source domain and target domain are not related to each other, thus a brute-force transfer may be highly unsuccessful. In the worst case, it may even degrade the performance of the target domain, a situation referred to as negative transfer (for example, when an Indian or British tourist in Europe learns to drive on the left side of the road).

The advantages of using transfer learning are clear: a change of features, domains, tasks, and probability distributions from the training to the testing phase does not require the underlying learning model to be rebuilt. The disadvantages are listed as follows:

- Most existing transfer learning algorithms assume that the source and target domains are related to each other in some sense. However, if the assumption does not hold, negative transfer may happen. In order to avoid negative transfer learning, measures to ascertain transferability between source domains and target domains needs to be studied. Based on this study of possible transferability, we can then select relevant source domains/tasks to extract knowledge for learning the target domains/tasks.

- Most existing transfer learning algorithms so far have focused on improving generalization across different distributions between source and target domains or tasks. In doing so, they assumed that the feature spaces between the source and target domains are the same. However, in many applications, we may wish to transfer knowledge across domains or tasks that have different feature spaces. This type of transfer learning is referred to as heterogeneous transfer learning, which remains a challenge yet to be overcome (Pan \& Yang (2010)).

A perfect example of transfer learning is the TRAMP (Transfer learning Action Models for Planning) system. TRAMP, given a set of traces in the target domain and expert-created domain models in the source domain, learns action models in the target domain by transferring knowledge from the source domain. TRAMP first encodes the input plan traces, which are state-action interleavings, into propositional formulas then as databases. Second, TRAMP encodes the action models from the source domains to a propositional formula set. Thirdly, 
TRAMP generates a candidate formula set to structure the target action models, building mappings between the source and target domains by searching keywords of predicates and actions from the Web then calculating similarities of Web pages to bridge the gap between the two and transfer knowledge. Finally, TRAMP learns action models from the transferred knowledge with the help of Markov Logic Networks (Zhuo \& Yang (2014)).

\subsection{MAX-SAT based approaches}

A weighted MAX-SAT (maximum satisfiability) problem can be stated as: given a collection $C$ of $m$ clauses $\left(C_{1}, \ldots, C_{m}\right)$ involving the disjunction of $n$ logical variables, with clause weights $w_{i}$, find a truth assignment of the logical variables that maximizes the total weight of the satisfied clauses in $C$ (Zhuo \& Yang (2014)). Among works that use MAX-SAT, the LAWS (Learn Action models with transferring knowledge from a related source domain via Web search) system (Zhuo et al. (2011)) makes use of action models already created beforehand in other source domains, to help learn models in the target domain. The target domain and a related source domain are bridged by searching web pages related to the two domains, and then building a mapping between them by means of a similarity function which calculates the similarity between their corresponding Web pages. The similarity is calculated using the Kullback-Leibler (KL) divergence. Based on the calculated similarity, a set of weighted constraints, called web constraints, are built. Other constraints such as state, action and plan constraints, are also built from traces in the target domain. All the above constraints are solved using a weighted MAX-SAT solver, and target domain action models are reconstructed from the satisfied clauses.

The ARMS (Action-Relation Modelling System) (Yang et al. (2007)) system learns action models from traces which consist of action sequences occasionally interleaved with intermediate states. The parsed predicates are encoded in the form of intra-operator constraints called information and action constraints respected by these predicates. Frequent action pairs in the traces are identified with the help of the apriori algorithm (Agrawal \& Srikant (1994)), which are then used to obtain a set of intra-operator plan constraints. These constraints are then solved with the help of a SAT solver. The constraints which amount to true are used to reconstruct the actions of the domain model. The process iterates until all actions are modeled.

The RIM (Refining Incomplete planning domain Models through plan traces) system (Zhuo et al. (2013)) enriches its partial model during the process of learning. It constructs sets of soft and hard constraints from incomplete models and plan traces. These constraints are again intraoperator and inter-operator ones that must be satisfied by atomic actions as well as macro-actions. The novelty of this approach is that it learns macro-actions as well. Macro-actions increase the accuracy of the incomplete model. These constraints are solved with the help of a SAT solver. The constraints which amount to true are used to obtain sets of macro-actions and refined action models.

The Lammas (Learning Models for Multi-Agent Systems) system (Zhuo et al. (2013)) learns action models from input plan traces in the same lines of ARMS, however extended to a multiagent setting. Lammas also encodes the input plan traces as constraints which fall into one of the following three categories: (1) correctness constraints imposed on the relationship between actions in plan traces to ensure that causal links in the plan traces is not broken, (2) action constraints encoding the actions based on STRIPS semantics and (3) coordination constraints encoded from an Agent Interaction Graph (AIG) describing inter-agent interactions. The learnt actions are represented in the MA-STRIPS language which is more adapted for a multi-agent setting (Brafman \& Domshlak (2008)). 


\subsection{Supervised Learning Based Approaches}

In (Mourão et al. (2008)), the authors learn the effects of an agent's actions given a set of actions and their preconditions, in a partially observable and noisy environment. The input to the learning mechanism uses a vector representation that encodes a description of the action being performed and the state at which the action is applied. The final input vector representing a particular action applied to a certain state has the form: (actions, objectindependent properties, object-dependent properties). The output vector has the form: (object-independent properties, object-dependent properties). The task is to learn the associations between action-precondition pairs and their effects, that is, rules of the form $(A, \operatorname{Pre}(A) \rightarrow E f f(A))$. The learning problem has a set of binary classification problems, and the perceptron is used as a classifier. The prediction consists of calculating the kernel matrix, and the kernel trick is used in the case of non-linearly separable data (Mourão et al. (2008)).

Another work by the same authors (Mourão et al. (2010)) is based on the same lines, except that a voted kernel perceptron learning model with a Disjunctive Normal Form (DNF) kernel $\left(K(x, y)=2^{\text {same }(x, y)}\right.$, where same $(x, y)$ is the number of bits with equal values in $x$ and $\left.y\right)$ is used, allowing the perceptron to run over the entire space of possible rules (Sadohara (2001)). Action and state information is encoded in a vector representation as input to the learning model, and resulting state changes are produced in the form of an output vector. A third approach by the same authors (Mourão et al. (2012)) decomposes the learning problem into two stages: learning implicit action models and progressively deriving explicit rules from the implicit models. Rules are extracted from classifiers based on the notion that more discriminative features will contribute more to the classifier's objective function. The classifiers use the 3-DNF kernel.

A very recent work by the same authors (Mourão (2014)) learns probabilistic planning operators from noisy traces. While the precedent approaches find the most probable outcome, this approach emphasizes on generating alternately less probable outcomes from noisy traces. The rule fragments (partial precondition and single effect predicates) derived from classifiers that only contributed to the preconditions provide for a source of alternative effects for the planning operator. An alternative rule is created by reusing the precondition from the planning operator, and by one-by-one combining the effects of the rule fragments. Each new effect is accepted if the resulting rule gives better $\mathrm{F}$-scores than the previous rule on the remaining training examples. Once all the rule fragments have been considered, we delete the training examples covered by the new rule as well as those fragments that contributed to the new rule. This process iterates until no further rules can be created.

\section{Applications and Open Issues}

This section is dedicated to highlight some of the feats of AP in terms of deployments in realworld applications serving actual needs. It then proceeds to account for some key issues which continue to plague the domain.

\subsection{Applications of $A P$}

Planning has found its place into an array of real world applications which range from human multi-drones search-and-rescue missions (Bevacqua et al. (2015)), single-operator multi-aircraft guidance (Strenzke \& Schulte (2011)) to military evacuation operations (Muñoz-Avila et al. (1999)).

Robotics is one of the most appealing application areas for AP. The fast-developing world of social robotics has cited planning and its ability to scale to real-world problems posed by robotics as a key issue to resolve. This is because most work done in AP is focused on action planning, whereas actions are not the only aspects that a robot reasons upon. Planning also 
needs to account for temporal, causal, resource and information dependencies between computational, perceptual, and actuation components (Di Rocco et al. (2013)). This is increasingly falling into place. For example, the ROBIL team in the DARPA robotics challenge (DRC) (www.darpa.mil/NewsEvents/Releases/2012/10/24.aspx) used hierarchical plans for runtime monitoring of resources (Cohen et al. (2015)). The DARPA Grand Challenge is pivoted on path planning for autonomous vehicles, thus is beyond the scope of this paper. All in all, these works are gradual steps towards conceptualizing autonomous systems.

Achieving autonomous systems is one of the most prominent challenges of AP. AI planners have been successfully used in a wide range of applications with the intent of contributing towards the creation of autonomous systems. However AP alone is not sufficient to achieve such kind of autonomy, and requires the amalgamation of perception, comprehension and projection capabilities in order to achieve contextual awareness leading to fully autonomous behavior (Endsley \& Garland (2000)). From the perspective of AI planning and within the scope of this paper, we present a list of non-exhaustive guidelines that a robotic system can follow in order to be proclaimed autonomous:

- Capability of Autonomous Exploration and Conservative Planning: a robot must be capable of observing its surroundings using onboard sensors, selecting from a set of candidate locations, planning a trajectory and autonomously navigating to the selected location (Gregory et al. (2016)). Conservative planning is a method that seeks to exploit the classical planning framework, while simultaneously recognizing the opportunities (unexpected events). These opportunities are then used to increase the plan utility during its execution (Cashmore et al. (2016a)).

- Persistent Autonomy: capable of planning long-term behaviour and performing activity over extended periods of time without human intervention (Cashmore et al. (2016b)).

- Interleaving with other tasks: This entails the combination of two types of planners: AI task planners that handle the high-level symbolic reasoning part and motion planners that plan the movements in space and check the geometrical feasibility of the plans output by the task planners (Ferrer-Mestres et al. (2015)). It also entails concurrent planning and execution abilities so that the robot can achieve the goal as quickly as possible.

AP techniques have also been used extensively in the field of space exploration, notably in satellite, rover and spacecraft missions. For example, the Deep Space One employed a constraintbased integrated temporal planner and resource scheduler which performed periodic planning to manage resources and develop plans to achieve goals in a timely manner (Muscettola et al. (1998), Pell et al. (1997)).

\subsection{Open Issues}

Despite the aforementioned joint advances in the fields of AP and ML, there remain some issues which continue to cloud the domain. Some of the key issues are highlighted and discussed below.

- Fully Automated Planning-boon or bane? The necessity of fully automated planning can be debated in a two-fold fashion:

- Is it rational to envision a fully automated learning-planning-execution system? Despite the usage of the most comprehensive and state-of-the-art ML techniques, there is a certain amount of user-provided domain knowledge which cannot be discounted; especially bearing in mind the direct correlation between the speed of learning and the amount of domain knowledge furnished. Thus even the highest level of automation cannot weed out the need and advantages of human intervention.

- Is a fully automated system needed at all? Even the most competent ML system is somewhat of questionable use if it is not designed to factor in human's desire of being able to control its surroundings and automated systems. Modern day users, aware of the 
fact that an ideal system is difficult to envision, prefer a system that can not only be useful in a real-life situation, but can also be at the mercy of the user, such that the user gets autonomy over the representation mechanism, language, kind of traces etc.

\section{- Ignored Aspects:}

- Re-usable knowledge: The cross domain transfer of knowledge from an existing domain to enrich the model learning process in an alien domain (similar to the source domain) with few training examples, is an approach which has come into light recently with a series of works in transfer learning (Pan \& Yang (2010)).

- Learning during plan execution: this refers to a situation where the expected and obtained system state after an action execution are not in accord with each other. It arises due to a flawed domain theory. It has been a major topic of neglect owing to the fact that a flawed domain theory is somewhat of an alien concept in classical planning. This has, however, been a topic of greater attention given a flurry of approaches which have been pivoted on Surprise Based Learning (SBL) (Ranasinghe \& Shen (2008)).

- Learning with the time dimension: Time plays an imperative role in most real life domains. For example, each dialogue in a Human-Robot Interaction (HRI) is composed of an utterance further accompanied by gestural, body and eye movements; all of them interleaved in a narrow time frame. These interactions may thus be represented by a time sequence, with the intent of learning the underlying action model. Barring some initial works in this area, time remains an interesting dimension to explore ((Zhang et al. (2015)), Guillame-Bert \& Crowley (2012)).

- Direct re-applicability of learned model: Direct re-use of a learned model by a planner continues to remain a concern. A model that has been learned by applying ML techniques is more often than not incomplete, more concretely: inadept to be fed to a planner to directly generate plans. It needs to be retouched and fine tuned by a domain expert in order to be reusable. This marks a stark incapability of prominently used machine learning techniques to be comprehensive, leaving scope for more research.

- Extension of classical planning to a full scope domain: The applicability of the aforementioned approaches, most of which have been tested on highly simplified toy domains and not in real scenarios, remains an issue to be addressed. As mentioned in section 2, classical planning is founded on restrictive assumptions and dwells in determinism. However, the real world is laced with unpredictability: a predicate might switch its value spontaneously, the world may have hidden variables, the exact impact of actions may be unpredictable and so on (Zimmermann \& Kambhampati (2003)). Thus, the application of a model learned on benchmark traces into the real world remains a point to ponder about. Barring a few works (Ortiz et al. (2013)), a fair share of algorithms that treat noisy traces are learning on benchmark traces which they have been "self-adulterated" to produce noisy ones. This can be seen as a means of a conscious steering of the learning algorithm towards a higher rate of learning, raising questions over their neutrality.

\section{Conclusion}

Automated Planning (AP) has been gaining steam ever since studies into human problem solving, operations research (primarily state space search) and theorem proving started gaining momentum. This is because the notion of a series of actions orchestrating the accomplishment of a goal is one that resonates amongst all these fields. However, depending on the domain characteristics, its constituent actions are difficult to quantify thus codify. Planners are now equipped with the capability to reverse engineer the signatures, preconditions and effects of the domain-applicable actions. This reverse engineering is achieved by capitalizing on several state-of-the-art and classical machine learning (ML) techniques. The article broadly classifies ML approaches based on several criteria, along with the merits and limitations of each approach. It 
then highlights some persisting open issues with the discussed approaches. It concludes that while a significant number of interesting techniques have been applied to highly controlled experimental setups and toy domains, their full-blown application to diverse and uncertain real world scenarios remains a topic of further research. 
Table 3: State of the art planning algorithms $(\mathrm{PDM}=$ Partial Domain Model, OCL = Object Centered Language, $\mathrm{D}=$ Deterministic, $\mathrm{P}=$ Probabilistic, $\mathrm{FO}=$ Fully Observable, $\mathrm{PO}=$ Partially Observable, CNF = Conjunctive Normal Form, PDL = Prodigy Description Language, MAXSAT = Maximum Satisfiability, PGM = Probabilistic Graphical Model, GDA = Goal Driven Autonomy, FOL = First Order Logic), Granularity $=\{+($ high $), i($ intermediate $)$, $-($ low $)\}$

\begin{tabular}{|c|c|c|c|c|c|c|c|c|}
\hline Algorithm & Input & Output/Language & $\begin{array}{l}\text { Granul } \\
\text { arity }\end{array}$ & Technique & Merits & Limitations & $\begin{array}{l}\text { Envir } \\
\text { onment }\end{array}$ & $\begin{array}{l}\text { Robust } \\
\text { to } \\
\text { Noise }\end{array}$ \\
\hline \multicolumn{9}{|c|}{ Inductive Learning Based Approaches } \\
\hline ERA & $\begin{array}{l}\text { Map of area } \\
\text { and error models } \\
\text { that determine how } \\
\text { robots actions will } \\
\text { behave in different } \\
\text { terrain conditions }\end{array}$ & $\begin{array}{l}\text { Action Models/ } \\
\text { Simulation based }\end{array}$ & - & $\begin{array}{l}\text { Regression tree } \\
\text { induction }\end{array}$ & $\begin{array}{l}\text { Ability to model } \\
\text { noise and predict } \\
\text { continuous variables } \\
\text { in data }\end{array}$ & $\begin{array}{l}\text { Cannot make } \\
\text { multi-variate } \\
\text { predictions }\end{array}$ & $\mathrm{D}, \mathrm{FO}$ & $\mathrm{Y}$ \\
\hline LOCM & $\begin{array}{l}\text { Action } \\
\text { Sequences }\end{array}$ & $\begin{array}{l}\text { Action Models/ } \\
\text { PDDL }\end{array}$ & $i$ & $\begin{array}{l}\text { Inductive learning } \\
\text { on state machine } \\
\text { representation } \\
\text { of objects. }\end{array}$ & $\begin{array}{l}\text { Does not } \\
\text { require } \\
\text { background } \\
\text { information }\end{array}$ & $\begin{array}{l}\text { (i) Induced models } \\
\text { may contain } \\
\text { detectable flaws } \\
\text { (ii) Static background } \\
\text { information not } \\
\text { analysed by system }\end{array}$ & $\mathrm{D}, \mathrm{FO}$ & $\mathrm{N}$ \\
\hline LOCM2 & $\begin{array}{l}\text { Action Sequences/ } \\
\text { Action Schema }\end{array}$ & $\begin{array}{l}\text { Action Models/ } \\
\text { PDDL }\end{array}$ & $i$ & $\begin{array}{l}\text { Allows many } \\
\text { possible solutions } \\
\text { to same problem, } \\
\text { further using } \\
\text { heuristic criteria } \\
\text { to select a } \\
\text { single solution. } \\
\end{array}$ & $\begin{array}{l}\text { Does not } \\
\text { require } \\
\text { background } \\
\text { information }\end{array}$ & $\begin{array}{l}\text { Does not work in case } \\
\text { of domains with: } \\
\text { (i) Dynamic many-many } \\
\text { relationships } \\
\text { (ii) Same object more } \\
\text { than once in } \\
\text { action arguments }\end{array}$ & $\mathrm{D}, \mathrm{FO}$ & $\mathrm{N}$ \\
\hline NLOCM & $\begin{array}{l}\text { Action Sequences/ } \\
\text { Action Schema }\end{array}$ & $\begin{array}{l}\text { Action } \\
\text { Models/PDDL }\end{array}$ & + & $\begin{array}{l}\text { Inductive Logic } \\
\text { Programming }\end{array}$ & $\begin{array}{l}\text { Requires only final plan cost } \\
\text { as additional information }\end{array}$ & $\begin{array}{l}\text { Not equipped to } \\
\text { handle complex domains }\end{array}$ & $\mathrm{D}, \mathrm{FO}$ & $\mathrm{N}$ \\
\hline OpMaker & $\begin{array}{l}\text { Partial Model, } \\
\text { Action } \\
\text { sequences }\end{array}$ & Operators/OCL & - & $\begin{array}{l}\text { Operator induction by } \\
\text { mixed initiative }\end{array}$ & $\begin{array}{l}\text { (i) Ease of operator } \\
\text { encoding } \\
\text { (ii) Useful for } \\
\text { non-experts }\end{array}$ & $\begin{array}{l}\text { Needs user input } \\
\text { for intermediate state } \\
\text { information }\end{array}$ & $\mathrm{D}, \mathrm{FO}$ & $\mathrm{Y}$ \\
\hline OPMaker2 & $\begin{array}{l}\text { PDM, Action } \\
\text { sequences/OCL }\end{array}$ & Operators & + & $\begin{array}{l}\text { Computes intermediate } \\
\text { states using } \\
\text { combination of } \\
\text { heuristics, inference } \\
\text { from PDM, training } \\
\text { tasks and solutions }\end{array}$ & $\begin{array}{l}\text { (i) Overcomes drawback } \\
\text { of Opmaker by negating } \\
\text { need for user input } \\
\text { for intermediate state } \\
\text { information } \\
\text { (ii) Does not require too } \\
\text { many examples }\end{array}$ & $\begin{array}{l}\text { Expert required } \\
\text { to transfer heuristic } \\
\text { knowledge }\end{array}$ & $\mathrm{D}, \mathrm{FO}$ & Y \\
\hline PELA & $\begin{array}{l}\text { Planning problem, } \\
\text { PDM and } \\
\text { action sequences }\end{array}$ & $\begin{array}{l}\text { Enriched action } \\
\text { model/PDDL }\end{array}$ & $i$ & TDIDT & $\begin{array}{l}\text { Based on } \\
\text { off-the-shelf planning } \\
\text { and learning components }\end{array}$ & $\begin{array}{l}\text { Assumes correct } \\
\text { initial action model }\end{array}$ & $\mathrm{P}, \mathrm{FO}$ & $\mathrm{N}$ \\
\hline
\end{tabular}




\begin{tabular}{|c|c|c|c|c|c|c|c|c|}
\hline $\begin{array}{l}\text { Newton } \\
\text { et al. }(2007)\end{array}$ & $\begin{array}{l}\text { Domain and } \\
\text { example problems }\end{array}$ & Macros/PDDL & + & Genetic Algorithms & $\begin{array}{l}\text { (i) Allow planning of } \\
\text { several steps at } \\
\text { a time } \\
\text { (ii) Capture action sequences } \\
\text { that help avoid troublesome } \\
\text { regions }\end{array}$ & $\begin{array}{l}\text { Macros, when } \\
\text { replaced by constituent } \\
\text { actions, often result } \\
\text { in longer plans }\end{array}$ & $\mathrm{D}, \mathrm{FO}$ & $\mathrm{N}$ \\
\hline \multicolumn{9}{|c|}{ Reinforcement Learning Based Approaches } \\
\hline LOPE & $\begin{array}{l}\text { Number of execution } \\
\text { cycles, possible action set }\end{array}$ & $\begin{array}{l}\text { Operators/ } \\
\text { Propositional } \\
\text { Logic }\end{array}$ & + & Reinforcement Learning & $\begin{array}{l}\text { Allows knowledge sharing } \\
\text { among agents, } \\
\text { increasing percentage of } \\
\text { successful plans }\end{array}$ & $\begin{array}{l}\text { Sensor differences } \\
\text { among agents causes } \\
\text { different ways of } \\
\text { perception thus } \\
\text { different biases towards } \\
\text { operator generation } \\
\end{array}$ & $\mathrm{D}, \mathrm{FO}$ & $\mathrm{N}$ \\
\hline $\begin{array}{l}\text { Safaei \& } \\
\text { Ghassem- } \\
\text { Sani }(2007)\end{array}$ & Action sequences & $\begin{array}{l}\text { Operators/FOL } \\
\text { without functions }\end{array}$ & + & Incremental Learning & $\begin{array}{l}\text { (i) Removes redundant } \\
\text { predicates in precondition } \\
\text { (ii) Doesnt need } \\
\text { prior knowledge }\end{array}$ & $\begin{array}{l}\text { Conditional effects } \\
\text { not supported in } \\
\text { learned operators }\end{array}$ & $\mathrm{P}, \mathrm{FO}$ & $\mathrm{N}$ \\
\hline \multicolumn{9}{|c|}{ Relational Reinforcement Learning (RRL) Based Approaches } \\
\hline MARLIE & $\begin{array}{l}\text { Episodes } \\
\text { (Action Sequences) }\end{array}$ & $\begin{array}{l}\text { Transition and } \\
\text { reward function/ } \\
\text { Expressive } \\
\text { relational } \\
\text { language } \\
\end{array}$ & + & $\begin{array}{l}\text { Learning Relational } \\
\text { Decision Trees }\end{array}$ & $\begin{array}{l}\text { Relational thus } \\
\text { widely applicable }\end{array}$ & $\begin{array}{l}\text { Decision tree not } \\
\text { restructured when new } \\
\text { examples appear }\end{array}$ & $\mathrm{P}, \mathrm{FO}$ & $\mathrm{N}$ \\
\hline \multicolumn{9}{|c|}{ Uncertainty Based Approaches } \\
\hline AMAN & Action Sequence & Operators/STRIPS & + & $\begin{array}{l}\text { PGM, } \\
\text { Reinforcement Learning }\end{array}$ & $\begin{array}{l}\text { No background knowledge } \\
\text { needed, can learn } \\
\text { directly from traces }\end{array}$ & $\begin{array}{l}\text { Model sampling } \\
\text { mechanism unclear }\end{array}$ & $\mathrm{D}, \mathrm{PO}$ & $\mathrm{Y}$ \\
\hline LAMP & $\begin{array}{l}\text { State-Action } \\
\text { Interleavings }\end{array}$ & Action Models/PDDL & + & $\begin{array}{l}\text { Markov } \\
\text { Logic } \\
\text { Network (MLN) } \\
\end{array}$ & $\begin{array}{l}\text { More expressive models with } \\
\text { quantifiers } \\
\text { and logical implications }\end{array}$ & $\begin{array}{l}\text { Looses efficiency } \\
\text { with increasing } \\
\text { domain size } \\
\end{array}$ & $\mathrm{D}, \mathrm{FO}$ & $\mathrm{N}$ \\
\hline $\begin{array}{l}\text { Pasula et al. } \\
(2007)\end{array}$ & $\begin{array}{l}\text { State-Action } \\
\text { Interleavings }\end{array}$ & $\begin{array}{l}\text { Probabilistic } \\
\text { STRIPS-like rules/ } \\
\text { STRIPS-like }\end{array}$ & $i$ & $\begin{array}{l}\text { Learn action structure, } \\
\text { action outcomes } \\
\text { and parameter estimation }\end{array}$ & $\begin{array}{l}\text { Allows learning of models } \\
\text { of more realistic worlds }\end{array}$ & $\begin{array}{l}\text { Rules cannot } \\
\text { be applied } \\
\text { in parallel }\end{array}$ & $\mathrm{P}, \mathrm{FO}$ & $\mathrm{N}$ \\
\hline \multicolumn{9}{|c|}{ Surprise Based Learning (SBL) Approaches } \\
\hline ARTUE & $\begin{array}{l}\text { Initial state, } \\
\text { goal state, } \\
\text { model }\end{array}$ & $\begin{array}{l}\text { Expectations, } \\
\text { discrepancies } \\
\text { and goals/PDDL+ }\end{array}$ & $i$ & GDA & $\begin{array}{l}\text { Integrates AI research } \\
\text { in planning, environment } \\
\text { monitoring, explanation, goal } \\
\text { generation, and } \\
\text { goal management. }\end{array}$ & $\begin{array}{l}\text { Cannot be applied } \\
\text { to complex } \\
\text { environments }\end{array}$ & $\mathrm{D}, \mathrm{FO}$ & $\mathrm{N}$ \\
\hline EXPO & $\begin{array}{l}\text { PDM, } \\
\text { traces of state sequences }\end{array}$ & $\begin{array}{l}\text { New preconditions, } \\
\text { effects, conditional } \\
\text { effects, operators, } \\
\text { attribute values/PDL }\end{array}$ & - & $\begin{array}{l}\text { Learning-by- } \\
\text { experimentation } \\
\text { for operator refinement }\end{array}$ & $\begin{array}{l}\text { (i) Learns conditional effects } \\
\text { (ii) Methods are goal-directed } \\
\text { and learning is incremental }\end{array}$ & $\begin{array}{l}\text { Rules learnt from } \\
\text { general to specific }\end{array}$ & $\mathrm{D}, \mathrm{FO}$ & $\mathrm{N}$ \\
\hline LGDA & $\begin{array}{l}\text { Initial state, } \\
\text { dummy goal, } \\
\text { dummy discrepancy, } \\
\text { policy }\end{array}$ & $\begin{array}{l}\text { Expectations, } \\
\text { discrepancies } \\
\text { and goals }\end{array}$ & $i$ & $\begin{array}{l}\text { Uses case-based } \\
\text { reasoning and intent } \\
\text { recognition in order } \\
\text { to build GDA agents } \\
\text { that learn from } \\
\text { demonstrations } \\
\end{array}$ & $\begin{array}{l}\text { Case-based reasoning }(\mathrm{CBR}) \\
\text { and Reinforcement } \\
\text { Learning (RL) }\end{array}$ & $\begin{array}{l}\text { Lacks capabilities } \\
\text { to learn explanations } \\
\text { of discrepancies }\end{array}$ & $\mathrm{D}, \mathrm{FO}$ & $\mathrm{N}$ \\
\hline
\end{tabular}




\begin{tabular}{|c|c|c|c|c|c|c|c|c|}
\hline LIVE & $\begin{array}{l}\text { Sequence of states, } \\
\text { actions and predictions }\end{array}$ & $\begin{array}{l}\text { Prediction rule } \\
\text { (condition, action } \\
\text { and precondition)/ } \\
\text { CNF of condition, } \\
\text { action and precondition } \\
\end{array}$ & $i$ & $\begin{array}{l}\text { Surprise and explanation } \\
\text { generation. Uses a set } \\
\text { of domain-dependent } \\
\text { search heuristics } \\
\text { during planning }\end{array}$ & $\begin{array}{l}\text { Hidden predicates can } \\
\text { also be determined. }\end{array}$ & $\begin{array}{l}\text { Exploration method is } \\
\text { brute force }\end{array}$ & $\mathrm{D}, \mathrm{FO}$ & $\mathrm{N}$ \\
\hline OBSERVER & $\begin{array}{l}\text { Action Sequences, practice } \\
\text { problems }\end{array}$ & $\begin{array}{l}\text { Operators/ } \\
\text { STRIPS-like }\end{array}$ & $i$ & $\begin{array}{l}\text { Conservative } \\
\text { specific-to-general } \\
\text { inductive generalization } \\
\text { process }\end{array}$ & $\begin{array}{l}\text { (i) Can find out } \\
\text { negated preconditions } \\
\text { (ii) Does not require } \\
\text { strong background } \\
\text { knowledge. }\end{array}$ & $\begin{array}{l}\text { May suffer } \\
\text { from incomplete } \\
\text { or incorrect } \\
\text { domain knowledge }\end{array}$ & $\mathrm{D}, \mathrm{FO}$ & $\mathrm{N}$ \\
\hline \multicolumn{9}{|c|}{ Transfer Learning Based Approaches } \\
\hline TRAMP & $\begin{array}{l}\text { predicate set, } \\
\text { small set of } \\
\text { plan traces T } \\
\text { from the target } \\
\text { domain, set of } \\
\text { action models from } \\
\text { source domain. }\end{array}$ & $\begin{array}{l}\text { Action models in } \\
\text { target domain } D_{t} / \\
\text { STRIPS }\end{array}$ & $i$ & Transfer Learning & $\begin{array}{l}\text { Minimum training examples } \\
\text { needed to learn } \\
\text { target action model }\end{array}$ & $\begin{array}{l}\text { Possibility of } \\
\text { negative transfer }\end{array}$ & $\mathrm{D}, \mathrm{FO}$ & $\mathrm{N}$ \\
\hline \multicolumn{9}{|c|}{ MAX-SAT Based Approaches } \\
\hline ARMS & $\begin{array}{l}\text { Action sequence } \\
\text { with partial/no } \\
\text { state information }\end{array}$ & Operators/STRIPS & $i$ & $\begin{array}{l}\text { Builds weighted } \\
\text { propositional satisfiability } \\
\text { problem and solves } \\
\text { it using weighted } \\
\text { MAX-SAT solver }\end{array}$ & $\begin{array}{l}\text { Can handle } \\
\text { cases when intermediate } \\
\text { state observations } \\
\text { are difficult to acquire }\end{array}$ & $\begin{array}{l}\text { (i) Cannot learn action } \\
\text { models with quantifiers } \\
\text { or implications } \\
\text { (ii) Cannot learn } \\
\text { complex action models }\end{array}$ & $\mathrm{D}, \mathrm{FO}$ & $\mathrm{N}$ \\
\hline LAWS & $\begin{array}{l}\text { Action models, } \\
\text { predicate set, } \\
\text { small set of } \\
\text { plan traces T } \\
\text { from the target } \\
\text { domain, set of } \\
\text { action models from } \\
\text { source domain. }\end{array}$ & $\begin{array}{l}\text { Action models in } \\
\text { target domain } D_{t} / \\
\text { STRIPS }\end{array}$ & $i$ & $\begin{array}{l}\text { Transfer Learning+ } \\
\text { KL divergence }\end{array}$ & $\begin{array}{l}\text { Web can be exploited } \\
\text { as knowledge source }\end{array}$ & $\begin{array}{l}\text { Possibility of } \\
\text { negative transfer }\end{array}$ & $\mathrm{D}, \mathrm{FO}$ & $\mathrm{N}$ \\
\hline Lammas & Action sequences & $\begin{array}{l}\text { Operators/ } \\
\text { MA-STRIPS }\end{array}$ & $i$ & $\begin{array}{l}\text { Builds inter-agent and } \\
\text { intra-agent constraints and } \\
\text { solves it using } \\
\text { weighted MAX-SAT solver }\end{array}$ & $\begin{array}{l}\text { Can capture interactions } \\
\text { between agents }\end{array}$ & Not tested exhaustively & $\mathrm{D}, \mathrm{FO}$ & $\mathrm{N}$ \\
\hline \multicolumn{9}{|c|}{ Supervised Learning Based Approaches } \\
\hline $\begin{array}{l}\text { Mourao } \\
\text { et al. }(2008)\end{array}$ & Actions and states & Operators/PPDDL & + & $\begin{array}{l}\text { Kernel perceptrons }+ \\
\text { sequential covering }\end{array}$ & $\begin{array}{l}\text { Can handle noisy } \\
\text { domains }\end{array}$ & $\begin{array}{l}\text { Cannot handle } \\
\text { incomplete observations }\end{array}$ & $\mathrm{P}, \mathrm{FO}$ & $\mathrm{Y}$ \\
\hline
\end{tabular}




\section{References}

Agrawal, R. and Srikant, R., 1994. Fast algorithms for mining association rules. In Proc. 20th int. conf. very large data bases, 487-499.

Balac, N., Gaines, D.M. and Fisher, D., 2000. Learning action models for navigation in noisy environments. In ICML Workshop on Machine Learning of Spatial Knowledge.

Bevacqua, G., Cacace, J., Finzi, A. and Lippiello, V., 2015. Mixed-Initiative Planning and Execution for Multiple Drones in Search and Rescue Missions. In ICAPS, 315-323.

Brafman, R.I. and Domshlak, C., 2008. From One to Many: Planning for Loosely Coupled Multi-Agent Systems. In ICAPS, 28-35.

Bresina, J. and Morris, P., 2007. Mixed-initiative planning in space mission operations. In AI magazine, $28(2), 75$.

Cashmore, M., Fox, M., Long, D., Ridder, B.C., and Magazzeni, D., 2016a. Opportunistic Planning for Increased Plan Utility. In Proceedings of the 4th ICAPS Workshop on Planning and Robotics (PlanRob 2016), 82-92.

Cashmore, M., Fox, M., Long, D., and Ridder, B.C., and Magazzeni, D., 2016b. Strategic Planning for Autonomous Systems over Long Horizons. In Proceedings of the 4 th ICAPS Workshop on Planning and Robotics (PlanRob 2016), 74-81.

Cohen, L., Shimony, S.E. and Weiss, G., 2015. Estimating the probability of meeting a deadline in hierarchical plans. In Computational Logic in Multi-Agent Systems, 243-258.

Cresswell, S. and Gregory, P., 2011. Generalised Domain Model Acquisition from Action Traces. In International Conference on Automated Planning and Scheduling.

Cresswell, S.N., McCluskey, T.L. and West, M.M., 2009. Acquisition of object-centered domain models from planning examples. In International Conference on Automated Planning and Scheduling, 338-341.

Cresswell, S.N., McCluskey, T.L. and West, M.M., 2013. Acquiring planning domain models using LOCM. In The Knowledge Engineering Review, 28(02), 195-213.

Croonenborghs, T., Ramon, J., Blockeel, H. and Bruynooghe, M., 2007, January. Online Learning and Exploiting Relational Models in Reinforcement Learning. In International Joint Conference on Artificial Intelligence, 726-731.

De Jonge, F., Roos, N. and Witteveen, C., 2009. Primary and secondary diagnosis of multi-agent plan execution. In Autonomous Agents and Multi-Agent Systems, 18(2), 267-294.

Deshpande, A., Milch, B., Zettlemoyer, L.S. and Kaelbling, L., 2007. Learning Probabilistic Relational Dynamics for Multiple Tasks. Probabilistic, Logical and Relational Learning - A Further Synthesis.

Di Rocco, M., Pecora, F. and Saffiotti, A., 2013. Closed loop configuration planning with time and resources. In Planning and Robotics, 36.

Driessens, K., Ramon, J. and Blockeel, H., 2001. Speeding up relational reinforcement learning through the use of an incremental first order decision tree learner. In European Conference on Machine Learning, 97-108.

Dz̃eroski, S., De Raedt, L. and Driessens, K., 2001. Relational reinforcement learning. In Machine learning, 43(1-2), 7-52.

Endsley, M.R. and Garland, D.J., 2000. Theoretical underpinnings of situation awareness: A critical review. In Situation awareness analysis and measurement, 1, 3-32.

Fikes, R.E. and Nilsson, N.J., 1971. STRIPS: A new approach to the application of theorem proving to problem solving. In Artificial intelligence, 2(3-4), 189-208. 
Ferrer-Mestres, J., Frances, G. and Geffner, H., 2015. Planning with state constraints and its application to combined task and motion planning. In Proc. of Workshop on Planning and Robotics (PLANROB), $13-22$.

García-Martínez, R. and Borrajo, D., 2000. An integrated approach of learning, planning, and execution. In Journal of Intelligent and Robotic Systems, 29(1), 47-78.

Ghallab, M., Nau, D. and Traverso, P., 2004. Automated planning: theory \& practice. Elsevier.

Gil, Y., 1992. Acquiring domain knowledge for planning by experimentation, PhD. thesis, Dept of Computer Science, Carnegie-Mellon University.

Guillame-Bert, M. and Crowley, J.L., 2012. Learning Temporal Association Rules on Symbolic Time Sequences. In Asian Conference on Machine Learning, 159-174.

Gregory, P. and Cresswell, S., 2015. Domain Model Acquisition in the Presence of Static Relations in the LOP System. In ICAPS, 97-105.

Gregory, J., Fink, J., Rogers, J., and Gupta, S., and Crowley, J.L., 2016. A Risk-Based Framework for Incorporating Navigation Uncertainty Into Exploration Strategies. In Proceedings of the 4th ICAPS Workshop on Planning and Robotics (PlanRob 2016), 176-183.

Gregory, P. and Lindsay, A., 2016. Domain model acquisition in domains with action costs. In Proceedings of the Twenty-Sixth International Conference on International Conference on Automated Planning and Scheduling, 149-157.

Inoue, K., Ribeiro, T. and Sakama, C., 2014. Learning from interpretation transition. In Machine Learning, 94(1), 51-79.

Jaidee, U., Muñoz-Avila, H. and Aha, D.W., 2011. Integrated learning for goal-driven autonomy. In IJCAI, 22(3), 2450.

Jilani, R., Crampton, A., Kitchin, D.E. and Vallati, M., 2014. Automated knowledge engineering tools in planning: state-of-the-art and future challenges. In Knowledge Engineering for Planning and Scheduling.

Jilani, R., Crampton, A., Kitchin, D. and Vallati, M., 2015. ASCoL: A tool for improving automatic planning domain model acquisition. In Congress of the Italian Association for Artificial Intelligence, 438-451.

Jiménez, S., De La Rosa, T., Fernández, S., Fernández, F. and Borrajo, D., 2012. A review of machine learning for automated planning. In The Knowledge Engineering Review, 27(04), 433-467.

Jiménez, S., Fernández, F. and Borrajo, D., 2008. The PELA architecture: integrating planning and learning to improve execution. In Association for the Advancement of Artificial Intelligence.

Kalech, M., 2012. Diagnosis of coordination failures: a matrix-based approach. In Autonomous Agents and Multi-Agent Systems, 24(1), 69-103.

Mart'nez, D., Alenya, G., Torras, C., Ribeiro, T. and Inoue, K., 2016. Learning relational dynamics of stochastic domains for planning. In Proceedings of the 26th International Conference on Automated Planning and Scheduling.

McCluskey, T.L., Cresswell, S.N., Richardson, N.E. and West, M.M., 2009. Automated acquisition of action knowledge.

McCluskey, T.L., Richardson, N.E. and Simpson, R.M., 2002. An Interactive Method for Inducing Operator Descriptions. In Artificial Intelligence Planning Systems, 121-130.

McDermott, D., Ghallab, M., Howe, A., Knoblock, C., Ram, A., Veloso, M., Weld, D. and Wilkins, D., 1998. PDDL-the planning domain definition language. 
Micalizio, R. and Torasso, P., 2014. Cooperative Monitoring to Diagnose Multiagent Plans. J. Artif. Intell. Res.(JAIR), 51, 1-70.

Molineaux, M. and Aha, D.W., 2014. Learning unknown event models. In Association for the Advancement of Artificial Intelligence, 395-401.

Molineaux, M., Klenk, M. and Aha, D.W., 2010. Goal-driven autonomy in a Navy strategy simulation. KNEXUS RESEARCH CORP SPRINGFIELD VA.

Mourão, K., 2014. Learning probabilistic planning operators from noisy observations. In Proc. of the Workshop of the UK Planning and Scheduling Special Interest Group.

Mourão, K., Petrick, R.P. and Steedman, M., 2008. Using kernel perceptrons to learn action effects for planning. In International Conference on Cognitive Systems (CogSys 2008), 45-50.

Mourão, K., Petrick, R.P. and Steedman, M., 2010. Learning action effects in partially observable domains. In European Conference on Artificial Intelligence, 973-974.

Mourão, K., Zettlemoyer, L.S., Petrick, R. and Steedman, M., 2012. Learning STRIPS Operators from Noisy and Incomplete Observations. In Proceedings of the Twenty-Eighth Conference on Uncertainty in Artificial Intelligence, 614-623.

Mourão, K., 2012. Learning action representations using kernel perceptrons (Doctoral Dissertation, The University of Edinburgh).

Muñoz-Avila, H., Aha, D.W., Breslow, L. and Nau, D., 1999. HICAP: An interactive case-based planning architecture and its application to noncombatant evacuation operations. In Proceedings of the sixteenth national conference on Artificial intelligence and the eleventh Innovative applications of artificial intelligence conference innovative applications of artificial intelligence, 870-875.

Muscettola, N., Nayak, P.P., Pell, B. and Williams, B.C., 1998. Remote agent: To boldly go where no AI system has gone before. In Artificial Intelligence, 103(1-2), 5-47.

Newton, M.A.H. and Levine, J., 2010. Implicit Learning of Compiled Macro-Actions for Planning. In European Conference on Artificial Intelligence, 323-328.

Newton, M.A.H., Levine, J., Fox, M. and Long, D., 2007. Learning Macro-Actions for Arbitrary Planners and Domains. In International Conference on Automated Planning and Scheduling, 256-263.

Newton, M.H., Levine, J., Fox, M. and Long, D., 2008. Learning macros that are not captured by given example plans. In Poster Papers at the International Conference on Automated Planning and Scheduling.

Nilsson, N.J., 1984. Shakey the robot. SRI INTERNATIONAL MENLO PARK CA.

Ortiz, J., Garca-Olaya, A. and Borrajo, D., 2013. Using Roni Stern for building planning action models. In International Journal of Distributed Sensor Networks, 9(6), 942347.

Pan, S.J. and Yang, Q., 2010. A survey on transfer learning. In IEEE Transactions on knowledge and data engineering, 22(10), 1345-1359.

Pasula, H., Zettlemoyer, L.S. and Kaelbling, L.P., 2004. Learning Probabilistic Relational Planning Rules. In International Conference on Automated Planning and Scheduling, 73-82.

Pasula, H., Zettlemoyer, L.S. and Kaelbling, L.P., 2007. Learning symbolic models of stochastic domains. In Journal of Artificial Intelligence Research, 309-352.

Pednault, E.P., 1989. ADL: Exploring the Middle Ground Between STRIPS and the Situation Calculus. In $K r, 89,324-332$.

Pell, B., Gat, E., Keesing, R., Muscettola, N. and Smith, B., 1997, August. Robust periodic planning and execution for autonomous spacecraft. In IJCAI, 1234-1239. 
Quinlan, J.R., 1986. Induction of decision trees. In Machine learning, 1(1), 81-106.

Ranasinghe, N. and Shen, W., 2008, August. Surprise-based learning for developmental robotics. In Learning and Adaptive Behaviors for Robotic Systems, 65-70.

Richardson, M. and Domingos, P., 2006. Markov logic networks. In Machine learning, 62(1-2), 107-136.

Rodrigues, C., Gérard, P., Rouveirol, C. and Soldano, H., 2011. Active learning of relational action models. In International Conference on Inductive Logic Programming, 302-316.

Sadohara, K., 2001. Learning of boolean functions using support vector machines. In International Conference on Algorithmic Learning Theory, 106-118.

Safaei, J. and Ghassem-Sani, G., 2007. Incremental learning of planning operators in stochastic domains. In International Conference on Current Trends in Theory and Practice of Computer Science, 644-655.

Sanner, S., 2010. Relational dynamic influence diagram language (rddl): Language description. Unpublished ms. Australian National University.

Shah, M., Chrpa, L., Jimoh, F., Kitchin, D., McCluskey, T., Parkinson, S. and Vallati, M., 2013. Knowledge engineering tools in planning: State-of-the-art and future challenges. Knowledge Engineering for Planning and Scheduling, 53, 53.

Shen, W.M., 1993. Discovery as autonomous learning from the environment. In Machine Learning, 12(1-3), 143-165.

Silver, D., Huang, A., Maddison, C.J., Guez, A., Sifre, L., Van Den Driessche, G., Schrittwieser, J., Antonoglou, I., Panneershelvam, V., Lanctot, M. and Dieleman, S., 2016. Mastering the game of Go with deep neural networks and tree search. In Nature, 529(7587), 484-489.

Strenzke, R. and Schulte, A., 2011. The MMP: A mixed-initiative mission planning system for the multi-aircraft domain. In Proceeding of the International Conference on Automated Planning and Scheduling, 74-82.

Stern, R. and Brendan, J., 2017. Efficient, Safe, and Probably Approximately Complete Learning of Action Models. In IJCAI.

Stulp, F., Fedrizzi, A., Mösenlechner, L. and Beetz, M., 2012. Learning and reasoning with action-related places for robust mobile manipulation. In Journal of Artificial Intelligence Research, 43, 1-42.

Sutton, R.S. and Barto, A.G., 1998. Reinforcement learning: An introduction. MIT press.

Walsh, T.J. and Littman, M.L., 2008. Efficient Learning of Action Schemas and Web-Service Descriptions. In Association for the Advancement of Artificial Intelligence, 714-719.

Wang, X., 1996. Learning planning operators by observation and practice (Doctoral dissertation, Carnegie Mellon University).

Weber, B.G., Mateas, M. and Jhala, A., 2012. Learning from Demonstration for Goal-Driven Autonomy. In Association for the Advancement of Artificial Intelligence.

Winograd, T., 1972. Understanding natural language. In Cognitive psychology, 3(1), 1-191.

Yang, Q., Wu, K. and Jiang, Y., 2007. Learning action models from plan examples using weighted MAX-SAT. In Artificial Intelligence, 171(2-3), 107-143.

Yoon, S. and Kambhampati, S., 2007. Towards model-lite planning: A proposal for learning and planning with incomplete domain models. In ICAPS 2007 Workshop on Artificial Intelligence Planning and Learning.

Younes, H.L., Littman, M.L., Weissman, D. and Asmuth, J., 2005. The First Probabilistic Track of the International Planning Competition. J. Artif. Intell. Res.(JAIR), 24, 851-887. 
Zettlemoyer, L.S., Pasula, H. and Kaelbling, L.P., 2005. Learning planning rules in noisy stochastic worlds. In Association for the Advancement of Artificial Intelligence, 911-918.

Zhang, Y., Sreedharan, S. and Kambhampati, S., 2015. Capability models and their applications in planning. In Proceedings of the 2015 International Conference on Autonomous Agents and Multiagent Systems, 1151-1159.

Zhuo, H.H. and Kambhampati, S., 2013. Action-Model Acquisition from Noisy Plan Traces. In International Joint Conference on Artificial Intelligence.

Zhuo, H.H., Muñoz-Avila, H. and Yang, Q., 2011, May. Learning action models for multi-agent planning. In The 10th International Conference on Autonomous Agents and Multiagent Systems, 1, 217-224.

Zhuo, H.H., Nguyen, T.A. and Kambhampati, S., 2013. Refining Incomplete Planning Domain Models Through Plan Traces. In International Joint Conference on Artificial Intelligence.

Zhuo, H.H. and Yang, Q., 2014. Action-model acquisition for planning via transfer learning. In Artificial intelligence, 212, 80-103.

Zhuo, H.H., Yang, Q., Hu, D.H. and Li, L., 2010. Learning complex action models with quantifiers and logical implications. In Artificial Intelligence, 174(18), 1540-1569.

Zimmerman, T. and Kambhampati, S., 2003. Learning-assisted automated planning: looking back, taking stock, going forward. In AI Magazine, 24(2):73. 